\title{
WHITE NOISE HEISENBERG EVOLUTION AND EVANS-HUDSON FLOWS
}

\author{
LUIGI ACCARDI \\ Centro Vito Volterra, Università di Roma "Tor Vergata", \\ Via Columbia 2, 00133 Roma, Italy \\ accardi@volterra.mat.uniroma2.it \\ WIDED AYED \\ Institut Préparatoire aux Études d'Ingénieurs, \\ El Merezka, 8000, Nabeul, Tunisia \\ ayed_wided@yahoo.fr \\ HABIB OUERDIANE \\ Department of Mathematics, \\ Faculté des Sciences de Tunis, 1060, Tunis, Tunisia \\ habib.ouerdiane@fst.rnu.tn \\ Received 28 January 2005 \\ Revised 15 July 2005 \\ Communicated by H. H. Kuo
}

\begin{abstract}
We study white noise Heisenberg equations giving rise to flows which are *automorphisms of the observable algebra, but not necessarily inner automorphisms. We prove that the causally normally ordered form of these white noise Heisenberg equations are equivalent to Evans-Hudson flows. This gives in particular, the microscopic structure of the maps defining these flows, in terms of the original white noise derivations.
\end{abstract}

Keywords: Time consecutive principle; causal normal order; white noise stochastic calculus; quantum probability.

AMS Subject Classification: 60H40, 60H35, 46L55, 58J65

\section{Introduction}

\subsection{Statement of the problem}

The possibility to write stochastic differential equations as white noise Hamiltonian equations is one of the main advantages of white noise calculus with respect to stochastic calculus.

This approach evidences the role of regularization (through the choice of the constant in the causal commutation relations, cf. Sec. 2.2 below) which, in the 
classical case, is essentially equivalent to the choice of a notion of stochastic integral (Itô, Stratonovich, ...).

Moreover, the formal unitarity condition, for white noise equations, simply amounts to the formal self-adjointness of the white noise Hamiltonian, in agreement with the physical intuition. In the bounded case (the only one considered here), formal unitarity implies unitarity.

Usual Hamiltonian equations generate one-parameter unitary groups (unitary cocycles, in the interaction representation) and these define inner Heisenberg evolutions, i.e. inner one-parameter automorphism groups of the algebra of observables.

However, there are many important physical systems whose Heisenberg dynamics is not inner (e.g. infinite lattice systems), i.e. the generator of the dynamics is a *-derivation which is not expressible as the commutator with an element of the algebra of observables (an outer derivation).

Since white noise Hamiltonian equations generate inner Heisenberg evolutions, it is natural to ask oneself if one can introduce some non-inner white noise Heisenberg equations so to obtain non-inner dynamics.

In this paper we prove that the answer to the above question is affirmative and that the causally normally ordered form of a white noise Heisenberg equation (cf. Definition 2.2 below) is an Evans-Hudson flow. ${ }^{16,18}$ As a corollary we obtain the microscopic structure of the Evans-Hudson structure maps in terms of the original white noise derivation just as Accardi, Lu and Volovich obtained the microscopic structure of the coefficients of an Hudson-Parthasarathy equation in terms of the original white noise Hamiltonian.

In both cases the coefficients of the stochastic equations are nontrivial (in particular nonlinear) functions of the coefficients of the white noise equations and, while the latter coefficients have a direct physical interpretation, the former in general have not.

The plan of this paper is the following. In Sec. 1.2 we introduce some basic notations. In Sec. 2.1 we prove a weak form of the time consecutive principle which is sufficient for the purposes of this paper. This result is used, in Sec. 2.2 to extend to maps the original result obtained by Accardi, Lu and Volovich ${ }^{5}$ on the causal normal order of white noise equations and extended by J. Gough to more general (not necessarily causal) Stratonovich type white noise integrals. ${ }^{12,17}$

In Sec. 2.3, in order to clarify the difference between Schrödinger or Heisenberg evolutions and their white noise analogue, we recall some terminology concerning various types of Heisenberg evolutions: inner, outer, backward, forward, ....

In Sec. 2.4 we rewrite an inner white noise Heisenberg equation in such a way to suggest a natural outer generalization (cf. Definition 3.1). The problem here is to separate the outer part, which is entirely due to system operators, from the white noise part, which is inner.

Once this is done, using the time consecutive principle, we can put in causal normal order the white noise Heisenberg equation (cf. Sec. 3). 
At this point we can use the equivalence between stochastic equations and normally ordered white noise equations (equivalence principle). However, what we get with this equivalence is not yet an Evans-Hudson flow but its backward version. In Sec. 4 we deduce the structure equations for the homomorphic backward white noise Heisenberg equations.

On the other hand, using the equivalence principle, one can immediately write the normally ordered white noise equation associated to an Evans-Hudson flow (cf. Sec. 5). We prove that the homomorphism conditions for these flows coincide with the Evans-Hudson condition and with the homomorphism conditions for the backward Heisenberg evolutions.

We conjecture that there is a full identification of the Evans-Hudson flows with the causally normally ordered form of a white noise forward Heisenberg evolution. This problem will be discussed elsewhere. The difficulty lies in the fact that, the forward equations are more delicate than the backward: their causually normally ordered form cannot be deduced from the time consecutive principle without a series expansion (which is not discussed here).

Sections 6 and 7 are included to help the reader in bridging the outer and the, now well known, inner case. We have included a proof of the unitarity condition: here the difference between forward and backward evolution is reflected by the difference of the approaches needed to prove the isometry and the co-isometry conditions.

\subsection{Notations}

This paper is the second one of a series where we continue the program of giving a rigorous mathematical basis to the white noise approach to stochastic calculus. For this reason here we will keep notations to a minimum and we refer to Accardi, Ayed and Ouerdiane, ${ }^{6}$ for a detailed description of the quantities involved.

All operators here act on the Hilbert space $\mathcal{H}:=\mathcal{H}_{S} \otimes \Gamma$ where $\Gamma$ is the Boson Fock space over $L^{2}(\mathbb{R} ; \mathcal{K})$ and $\mathcal{K}, \mathcal{H}_{S}$ are Hilbert spaces (always complex separable unless explicitly stated) called respectively initial (or system) space and multiplicity (or polarization) space. The initial algebra $\mathcal{B}_{S}$ is a $C^{*}$-subalgebra of $\mathcal{B}\left(\mathcal{H}_{S}\right)$ (the algebra of all bounded operators on $\mathcal{H}_{S}$ ). The observable algebra $\mathcal{B}_{S}$ is $\mathcal{A}:=\mathcal{B}_{S} \otimes \mathcal{B}_{R}$ where $\mathcal{B}_{R}:=\mathcal{B}(\Gamma)$ is called the noise algebra and the tensor product is closed under the natural topology on $\mathcal{H}_{S} \otimes \Gamma$. We enlarge the noise algebra so as to include the polynomials, in the sense of distributions, in the field operators ${ }^{6}$ for a detailed description of this algebra and of the domain where it acts.

If $b_{S} \in \mathcal{B}_{S}$ and $b_{R} \in \mathcal{B}_{R}$, we will often write $b_{S} b_{R}$ instead of $b_{S} \otimes b_{R}$.

A bounded linear map $Z \mathcal{A}:=\mathcal{B}_{S} \otimes \mathcal{B}_{R} \rightarrow \mathcal{A}$ on the observable algebra is called of system type if it commutes with the noise, i.e. if it satisfies:

$$
Z\left(b_{R} x b_{R}^{\prime}\right)=b_{R} Z(x) b_{R}^{\prime} ; \quad x \in \mathcal{A} ; \quad b_{R}^{\prime}, b_{R} \in \mathcal{B}_{R} .
$$

This is equivalent to saying that $Z$ has the form $Z=\hat{Z} \otimes \mathrm{id}_{\mathcal{B}_{R}}$ for some linear map $Z: \mathcal{B}_{S} \rightarrow \otimes \mathcal{B}_{S}$. Noise type linear maps are defined analogously. Notice that, if $\alpha_{\varepsilon}$ 
is a system type map and $X_{R}$ is a noise type map, then

$$
\alpha_{\varepsilon} \circ X_{R}=X_{R} \alpha_{\varepsilon} .
$$

\section{Causal Normal Ordering}

\subsection{The time consecutive principle}

Definition 2.1. Let

$$
D_{\varepsilon}= \begin{cases}A, & \varepsilon=-1, \\ B, & \varepsilon=+1, \\ C, & \varepsilon=0 \\ T, & \varepsilon=2 .\end{cases}
$$

be

(i) either bounded linear operators on the initial space $\mathcal{H}_{S}$,

(ii) or bounded linear maps of system type.

A solution of the white noise equation:

$$
\partial_{t} U_{t}=-i\left(A b_{t}+B b_{t}^{+}+b_{t}^{+} T b_{t}+C\right) U_{t}=b_{t}^{\varepsilon} D_{\varepsilon} U_{t}, \quad U_{0}=1,
$$

where

$$
b_{t}^{\varepsilon}= \begin{cases}b_{t}, & \varepsilon=-1, \\ b_{t}^{+}, & \varepsilon=+1, \\ 1, & \varepsilon=0, \\ b_{t}^{+} b_{t}, & \varepsilon=2,\end{cases}
$$

is a white noise adapted process $U_{t}$ (in the sense of Sec. 4 of Ref. 6 satisfying the identity:

$$
U_{t}=1-i \int_{0}^{t}\left(A b_{s}+B b_{s}^{+}+b_{s}^{+} T b_{s}+C\right) U_{s} d s=1-i \int_{0}^{t} b_{s}^{\varepsilon} D_{\varepsilon} U_{s} d s
$$

in the sense that the white noise integral on the right-hand side exists on the maximal algebraic domain $\mathcal{D}_{M A}$ and the identity holds.

Definition 2.2. A normally ordered form of Eq. (2.2) is an equation of the type

$$
\partial_{t} U_{t}=-i \hat{A} U_{t} b_{t}-i \hat{B} U_{t} b_{t}^{+}-i b_{t}^{+} \hat{T} U_{t} b_{t}-i \hat{C} U_{t}, \quad U_{0}=1
$$

which has the same solutions as (2.2). In case (i), if the symmetry conditions:

$$
A^{*}=B, \quad T=T^{*}, \quad C=C^{*}
$$

are satisfied, (2.2) is called a white noise Hamiltonian equation.

Remark. Notice that the symmetry conditions (2.6) are equivalent to the formal self-adjointness of the operator valued distribution: 


$$
H_{t}:=A b_{t}+B b_{t}^{+}+b_{t}^{+} T b_{t}+C .
$$

Such an operator valued distribution will be called a white noise Hamiltonian.

Remark. In Ref. 6, the authors obtained the estimates on the white noise integrals and used them to prove the existence and uniqueness theorem and a priori bounds on the solutions, for white noise equations with bounded coefficients in the non-normally ordered case. Once these estimates are given and keeping the boundedness assumption, the existence proof is based on routine arguments on the iterated series and can be extended without difficulties to the case (ii) and to non-normally ordered equations. In the following we will freely use such extensions without spelling out, due to space constraint, the simple modifications required in the two above-mentioned cases.

The normal order problem consists in giving prescriptions which, given an equation of the form (2.2), allow to write it in the form (2.5) and to explicitly compute the new coefficients $\hat{A}, \hat{B}, \hat{T}, \hat{C}$.

Definition 2.3. The pair $\left\{b_{t}^{+}, b_{t}\right\}$ is said to satisfy the causal commutation relations with parameter $\gamma_{-} \in \mathbb{C}$

$$
\operatorname{Re} \gamma_{-}>0
$$

if $\forall t$, for any pair of white noise adapted processes $F_{s}, G_{s}$ and for any $x_{s}, y_{s} \in$ $\left\{b_{s}, b_{s}^{+}, 1\right\}$ :

$$
\int_{0}^{t} x_{s} F_{s}\left[b_{t}, b_{s}^{+}\right] G_{s} y_{s} d s=\gamma_{-} \int_{0}^{t} x_{s} F_{s} \delta_{+}(t-s) G_{s} y_{s} d s=\gamma_{-} x_{t} F_{t} G_{t} y_{t} .
$$

Moreover, all the remaining commutators are zero under the integral sign. In this case we write:

$$
\left[b_{s}, b_{t}^{+}\right]=\gamma_{-} \delta_{+}(t-s) ; \quad \forall s, t \in \mathbb{R} ; s<t .
$$

Remark. For the origins of the causal commutation relations (2.9) we refer to Accardi, Lu and Volovich. ${ }^{1}$ The meaning of the symbol $\delta_{+}(t-s)$ is defined in Sec. IX of Ref. 5. In this paper we will use this symbol only in the identity (2.8) which can therefore be considered as a definition of the right-hand side of (2.9).

The Hudson-Parthasarathy calculus corresponds to the choice:

$$
\gamma_{-}=\frac{1}{2}
$$

In the following, we will write Eq. (2.2) in the form

$$
\partial_{t} U_{t}=b_{t}^{\varepsilon} \dot{U}_{\varepsilon, t}=b_{t}^{\varepsilon} D_{\varepsilon} U_{t}, \quad U_{0}=1
$$


where summation over the repeated index $\varepsilon \in\{-1,0,+1,2\}$ is understood and where, by definition

$$
\dot{U}_{-1, s}=-i A U_{s} ; \quad \dot{U}_{+1, s}=-i B U_{s} ; \quad \dot{U}_{0, s}=-i C U_{s} ; \quad \dot{U}_{2, s}=-i T U_{s} .
$$

Theorem 2.1. (Time consecutive principle: weak form) Let $U_{t}$ be the unique solution of the white noise equation (2.10) with bounded coefficients $D_{\varepsilon}$. Then, for any $t \in \mathbb{R}_{+}$and any $\varepsilon, \theta \in\{ \pm 1,0,2\}$

$$
\int_{0}^{t} b_{s}^{\varepsilon}\left[b_{t}^{\theta}, \dot{U}_{\varepsilon, s}\right] d s=\int_{0}^{t} D_{\varepsilon} b_{s}^{\varepsilon}\left[b_{t}^{\theta}, U_{s}\right] d s=0 .
$$

Proof. Clearly (2.12) will follow from

$$
\int_{0}^{t} b_{t_{1}}^{\varepsilon}\left[b_{t}^{\theta}, U_{t_{1}}\right] d t_{1}=0 ; \quad \forall \varepsilon, \theta \in\{ \pm 1,0,2\}
$$

and for $U_{t}$ the unique solution of $(2.10)$. Since $\left[b_{t}^{\theta}, 1\right]=0,(2.13)$ is equivalent to

$$
\begin{aligned}
\int_{0}^{t} b_{t_{1}}^{\varepsilon} \int_{0}^{t_{1}}\left[b_{t}^{\theta}, b_{t_{2}}^{\varepsilon_{1}} D_{\varepsilon} U_{t_{2}}\right] d t_{1} d t_{2} \\
=\int_{0}^{t} d t_{1} \int_{0}^{t_{1}} d t_{2} \cdots \int_{0}^{t_{n-1}} d t_{n} b_{t_{1}}^{\varepsilon}\left[b_{t}^{\theta}, D_{\varepsilon_{2}} \cdots D_{\varepsilon_{n}} b_{t_{2}}^{\varepsilon_{2}} \cdots b_{t_{n}}^{\varepsilon_{n}} U_{t_{n}}\right] \\
=D_{\varepsilon_{2}} \cdots D_{\varepsilon_{n}} \int_{\Delta_{n}^{t}} d t_{1} \cdots d t_{n} b_{t_{1}}^{\varepsilon}\left[b_{t}^{\theta}, b_{t_{2}}^{\varepsilon_{1}} \cdots b_{t_{n}}^{\varepsilon_{n}}\right] U_{t_{n}} \\
\quad+D_{\varepsilon_{2}} \cdots D_{\varepsilon_{n}} \int_{\Delta_{n}^{t}} d t_{1} \cdots d t_{n} b_{t_{1}}^{\varepsilon} b_{t_{2}}^{\varepsilon_{2}} \cdots b_{t_{n}}^{\varepsilon_{n}}\left[b_{t}^{\theta}, U_{t_{n}}\right]=: I_{n}+J_{n}
\end{aligned}
$$

The integral in $I_{n}$ is a sum of terms each of which contains a

$$
\delta_{+}\left(t-t_{j}\right) ; \quad j \geq 2 .
$$

From the theory of distributions on the standard simplex (see Ref. 5), we know that, since

$$
t \geq t_{1} \geq t_{2} \geq \cdots \geq t_{j}
$$

then any term containing $\delta_{+}\left(t-t_{j}\right)$ vanishes. Thus

$$
I_{n}=0 \text {. }
$$

Now consider the norm of the matrix element of the term $J_{n}$ with respect to two vectors $\varphi, \psi$ of the maximal algebraic domain. This is less than or equal to a finite sum of integrals of the form

$$
\int_{\Delta_{n}^{t}} d t_{1} \cdots d t_{n} f_{1}\left(t_{1}\right) \cdots f_{n}\left(t_{n}\right)\left\langle\xi,\left[b_{t}^{\theta}, U_{t_{n}}\right] \eta\right\rangle
$$


where $\xi \in J(\varphi), \eta \in J(\psi)$ (in the Accardi, Fagnola and Quaegebeur notations ${ }^{9}$ ). In particular both $\xi, \eta \in \mathcal{D}_{M A}$ and the $f_{1}, \ldots, f_{n}$ are positive functions that can be assumed to be bounded. Therefore $(2.15)$ is less than or equal to

$$
c^{n} \int_{\left(\Delta^{t}\right)_{n}} d t_{1} \cdots d t_{n}\left\{\left\|\left\langle b_{t}^{\theta+} \xi, U_{t_{n}} \eta\right\rangle\right\|+\left\|\left\langle\xi, U_{t_{n}} b_{t}^{\theta} \eta\right\rangle\right\|\right\} .
$$

Since $\xi, \eta \in \mathcal{D}_{M A}$, this term is a sum of terms of the form

$$
c^{n} f(t) \int_{\Delta_{n}^{t}} d t_{1} \cdots d t_{n}\left\|\left\langle\xi_{1}, U_{t_{n}} \eta\right\rangle\right\|
$$

or of the form

$$
c^{n} g(t) \int_{\Delta_{n}^{t}} d t_{1} \cdots d t_{n}\left\|\left\langle\xi, U_{t_{n}} \eta_{1}\right\rangle\right\|
$$

where $f, g$ are bounded functions, and $\xi_{1} \in J(\xi), \eta_{1} \in J(\eta)$.

But, from the a priori estimates on the solutions of Eq. (2.10) given in Theorem 5.1 of Ref. 6 , we know that, for $\xi^{\prime}, \eta^{\prime} \in \mathcal{D}_{M A}$ there exists a constant $c_{\xi^{\prime}, \eta^{\prime}}(t)$ such that:

$$
\left\|\left\langle\xi^{\prime}, U_{t} \eta^{\prime}\right\rangle\right\| \leq c_{\xi^{\prime}, \eta^{\prime}}(t) ; \quad \forall s \in[0, t] .
$$

Since, in our case, the $\xi^{\prime}, \eta^{\prime}$ can vary in a finite set, we finally obtain that $(2.15)$ is less than or equal to

$$
c_{\varphi, \psi}(t) c^{n} \int_{\Delta_{n}^{t}} d t_{1} \cdots d t_{n}=c_{\varphi, \psi}(t) \frac{(c t)^{n}}{n !} .
$$

Since this is true for any $n \in \mathbb{N}$, we conclude that the matrix element of the integrals

$$
\int_{0}^{t} b_{s}^{\varepsilon}\left[b_{t}^{\theta}, U_{s}\right] d s ; \quad \forall \varepsilon, \theta \in\{ \pm 1,0,2\}
$$

are zero for any pair of vectors $\varphi, \psi \in \mathcal{D}_{M A}$ and this proves (2.12).

\subsection{Causal normal order of white noise equations}

Corollary 2.1. If $U_{t}$ is a solution of Eq. (2.2), then one has:

$$
\begin{gathered}
{\left[b_{t}, U_{t}\right]=-i \gamma_{-}\left(B U_{t}+T b_{t} U_{t}\right),} \\
{\left[b_{t}^{+}, U_{t}^{*}\right]=-i \bar{\gamma}_{-}\left(U_{t}^{*} B^{*}+U_{t}^{*} b_{t}^{+} T^{*}\right),} \\
{\left[b_{t}, U_{t}^{*}\right]=i \gamma_{-} U_{t}^{*} A^{*}+i \gamma_{-} U_{t}^{*} b_{t} T^{*},} \\
{\left[b_{t}^{+}, U_{t}\right]=i \bar{\gamma}_{-} A U_{t}+i \bar{\gamma}_{-} T b_{t}^{+} U_{t} .}
\end{gathered}
$$




\section{Proof.}

$$
\begin{aligned}
{\left[b_{t}, U_{t}\right]=} & {\left[b_{t}, 1-i \int_{0}^{t}\left(A b_{s}+b_{s}^{+} B+b_{s}^{+} T b_{s}+C\right) U_{s} d s\right] } \\
= & -i A \int_{0}^{t}\left[b_{t}, b_{s} U_{s}\right] d s-i B \int_{0}^{t}\left[b_{t}, b_{s}^{+} U_{s}\right] d s-i T \int_{0}^{t}\left[b_{t}, b_{s}^{+} b_{s} U_{s}\right] d s \\
& -i C \int_{0}^{t}\left[b_{t}, U_{s}\right] d s .
\end{aligned}
$$

Developing the commutators with the Leibnitz rule and using Theorem 2.1 we see that this is equal to

$$
\begin{gathered}
-i B \int_{0}^{t}\left[b_{t}, b_{s}^{+}\right] U_{s} d s-i T \int_{0}^{t}\left[b_{t}, b_{s}^{+}\right] b_{s} U_{s} d s \\
=-i \gamma_{-} B \int_{0}^{t} \delta_{+}(t-s) U_{s} d s-i \gamma_{-} T \int_{0}^{t} \delta_{+}(t-s) b_{s} U_{s} d s \\
=-i \gamma_{-}\left(B U_{t}+T b_{t} U_{t}\right), \\
{\left[b^{+}, U\right]=-i A \int_{0}^{t}\left[b_{t}^{+}, b_{s}\right] U_{s} d s-i T \int_{0}^{t} b_{s}^{+}\left[b_{t}^{+}, b_{s}\right] u_{s} d s=i \gamma_{-} A U_{t}+i \gamma_{-} T b_{t}^{+} U_{t},} \\
b_{t}^{+} U_{t}=U_{t} b_{t}^{+}+i \bar{\gamma}_{-} A U_{t}+i \bar{\gamma}_{-} T b_{t}^{+} U_{t}, \\
\left(1-i \bar{\gamma}_{-} T\right) b_{t}^{+} U_{t}=U_{t} b_{t}^{+}+i \bar{\gamma}_{-} A U_{t}
\end{gathered}
$$

and this proves $(2.16)$. Similar arguments applied to the commutator $\left[b_{t}, U_{t}^{*}\right]$ lead to:

$$
\begin{aligned}
{\left[b_{t}, U_{t}^{*}\right]=} & {\left[b_{t}, 1+i \int_{0}^{t} U_{s}^{*}\left(b_{s}^{+} A^{*}+B^{*} b_{s}+b_{s}^{+} T^{*} b_{s}+C^{*}\right) d s\right] } \\
= & i \int_{0}^{t}\left[b_{t}, U_{s}^{*} b_{s}^{+}\right] A^{*} d s+i \int_{0}^{t}\left[b_{t}, U_{s}^{*} B^{*} b_{s}\right] d s \\
& +i \int_{0}^{t}\left[b_{t}, U_{s}^{*} b_{s}^{+} T^{*} b_{s}\right] d s+i \int_{0}^{t}\left[b_{t}, U_{s}^{*} C^{*}\right] d s \\
= & i \int_{0}^{t} U_{s}^{*}\left[b_{t}, b_{s}^{+}\right] A^{*} d s+i \int_{0}^{t} U_{s}^{*}\left[b_{t}, b_{s}^{+}\right] T^{*} b_{s} d s \\
= & i \gamma_{-} \int_{0}^{t} \delta_{+}(t-s) U_{s}^{*} A^{*} d s+i \gamma_{-} \int_{0}^{t} \delta_{+}(t-s) U_{s}^{*} T^{*} b_{s} d s \\
= & i \gamma_{-} U_{t}^{*} A^{*}+i \gamma_{-} U_{t}^{*} b_{t} T^{*}
\end{aligned}
$$

and this proves (2.18). The remaining two identities are proved by taking the adjoint. 
Corollary 2.2. In the above notations, if $\left(1+i \gamma_{-} T\right)$ and $\left(1+i \gamma_{-} T^{*}\right)$ are invertible, then denoting:

$$
K=\left(1+i \gamma_{-} T\right)^{-1} ; \quad K^{\prime}=\left(1+i \gamma_{-} T^{*}\right)
$$

one has

$$
\begin{gathered}
b_{t} U_{t}=-i \gamma_{-} K B U_{t}+K U_{t} b_{t}, \\
U_{t} b_{t}^{+}=-i \bar{\gamma}_{-} A U_{t}+K^{*} b_{t}^{+} U_{t} .
\end{gathered}
$$

Proof. From Corollary 2.1 one deduces that:

$$
b_{t} U_{t}=U_{t} b_{t}-i \gamma_{-}\left(B U_{t}+T b_{t} U_{t}\right)
$$

or equivalently

$$
\left(1+i \gamma_{-} T\right) b_{t} U_{t}=-i \gamma_{-} B U_{t}+U_{t} b_{t}
$$

and this proves (2.21). Similarly

$$
b_{t} U_{t}^{*}=U_{t}^{*} b_{t}+i \gamma_{-} U_{t}^{*} A^{*}+i \gamma_{-} U_{t}^{*} b_{t} T^{*}=i \gamma_{-} U_{t}^{*} A^{*}+U_{t}^{*} b_{t}\left(1+i \gamma_{-} T^{*}\right)
$$

or equivalently

$$
b_{t} U_{t}^{*}=i \gamma_{-} U_{t}^{*} A^{*}+U_{t}^{*} b_{t} K^{\prime}
$$

and this proves $(2.22)$.

\subsection{Forward and backward evolutions}

A forward Heisenberg evolution is a two-parameter family of automorphisms of $\mathcal{A}$

$$
j_{s, t}^{+}: \mathcal{A} \rightarrow \mathcal{A}, \quad s \leq t, s, t \in \mathbb{R}
$$

satisfying

$$
\begin{aligned}
j_{r, s}^{+} \circ j_{s, t}^{+} & =j_{r, t}^{+}, \quad r \leq s \leq t \\
j_{s, s}^{+} & =\operatorname{id}_{\mathcal{A}} .
\end{aligned}
$$

The inverse of a forward Heisenberg evolution

$$
j_{s, t}^{-}:=\left(j_{s, t}^{+}\right)^{-1}
$$

is called a backward Heisenberg evolution. It satisfies

$$
\begin{aligned}
j_{s, t}^{-} \circ j_{r, s}^{-} & =j_{r, t}^{-}, \quad r \leq s \leq t \\
j_{s, s}^{-} & =\operatorname{id}_{\mathcal{A}} .
\end{aligned}
$$

A smooth forward Heisenberg evolution satisfies an equation of the form

$$
\partial_{t} j_{s, t}^{+}=i j_{s, t}^{+} \circ \delta_{t}, \quad j_{s, s}^{+}=\operatorname{id}_{\mathcal{A}} ;
$$


where $i^{2}=-1$ and $\delta_{t}$ is a $*$-derivation on $\mathcal{A}$ (or on an appropriate subspace). The associated backward evolution satisfies the equation

$$
\partial_{t} j_{s, t}^{-}=-i \delta_{t} \circ j_{s, t}, \quad j_{s, s}^{-}=\mathrm{id}_{\mathcal{A}}
$$

A forward Heisenberg evolution $j_{s, t}^{+}$is called inner if each of the automorphisms $j_{s, t}^{+}$ is inner, i.e. if for any $s \leq t$ there exists a unitary operator $U_{s, t} \in \mathcal{A}$ such that

$$
j_{s, t}^{+}(x)=U_{s, t}^{*} x U_{s, t}, \quad x \in \mathcal{A} .
$$

The associated backward evolution $j_{s, t}^{-}$is also inner and satisfies

$$
j_{s, t}^{-}(x)=U_{s, t} x U_{s, t}^{*}, \quad x \in \mathcal{A} .
$$

One way to produce such evolutions is to start from a forward unitary operator evolution, i.e. a two-parameter family $U_{s, t}$ of unitary operators in $\mathcal{A}$ satisfying

$$
U_{s, t}^{+} U_{r, s}^{+}=U_{r, t}^{+}, \quad r \leq s \leq t,
$$

and to define $j_{s, t}^{+}$using (2.25).

If such an evolution is smooth, it satisfies an equation of the form

$$
\partial_{t} U_{s, t}^{+}=-i H_{t} U_{s, t}^{+}, \quad U_{s, s}^{+}=1 .
$$

The associated backward evolution is defined by

$$
U_{s, t}^{-}:=\left(U_{s, t}^{+}\right)^{*}
$$

through (2.26) and, in the smooth case, it satisfies the equation

$$
\partial_{t} U_{s, t}^{-}=U_{s, t}^{-} i H_{t}, \quad U_{s, s}^{-}=1 .
$$

For inner evolutions the Heisenberg equations (2.23), (2.24) become respectively

$$
\begin{aligned}
& \partial_{t} j_{s, t}^{+}(x)=j_{s, t}^{+}\left(i\left[x, H_{t}\right]\right), \quad j_{s, s}^{+}(x)=x \\
& \partial_{t} j_{s, t}^{-}(x)=i\left[H_{t}, j_{s, t}^{-}(x)\right],
\end{aligned}
$$

where $x \in \mathcal{A}$.

When $H_{t}$ is a self-adjoint operator, Eq. (2.27) is called a Schrödinger equation. When $H_{t}$ has the form

$$
H_{t}=A b_{t}^{+}+B b_{t}+T b_{t}^{+} b_{t}+C
$$

where $b_{t}^{ \pm}$is a boson Fock Brownian motion and $A, B, C, T$ are system operators satisfying

$$
A^{*}=B, \quad T^{*}=T, \quad C^{*}=C,
$$

then $(2.27)$ is called a white noise Hamiltonian equation. 


\subsection{Inner white noise Heisenberg evolutions}

Lemma 2.1. If $\{\cdot, \cdot\}$ denotes the anticommutator, then the following identities hold:

$$
\begin{aligned}
& \{a, x y\}=\{a, x\} y+x[y, a], \\
& \{a, x y\}=x\{y, a\}+[a, x] y .
\end{aligned}
$$

Proof. Equation (2.29) follows from the identity

$$
\{a, x y\}=a x y+x y a=\{a x y+x a y\}-x a y+x y a,
$$

and (2.30) follows from the identity:

$$
\{a, x y\}=a x y+x y a=x\{y a+a y\}-x a y+a x y .
$$

Recall that, in the notations (2.3), (2.1) a backward inner white noise Heisenberg equation has the form:

$$
\partial_{t} j_{t}(x)=\delta_{I, t}\left(j_{t}(x)\right)=i\left[H_{t}, j_{t}(x)\right]=\left[D_{\varepsilon} b_{t}^{\varepsilon}, j_{t}(x)\right]=\left[D_{\varepsilon}, j_{t}(x)\right] b_{t}^{\varepsilon}+D_{\varepsilon}\left[b_{t}^{\varepsilon}, j_{t}(x)\right] .
$$

But, since $D_{\varepsilon} b_{t}^{\varepsilon}=b_{t}^{\varepsilon} D_{\varepsilon}$, one also has

$$
\partial_{t} j_{t}(x)=\left[b_{t}^{\varepsilon} D_{\varepsilon}, j_{t}(x)\right]=\left[b_{t}^{\varepsilon}, j_{t}(x)\right] D_{\varepsilon}+b_{t}^{\varepsilon}\left[D_{\varepsilon}, j_{t}(x)\right] .
$$

Therefore, summing the two, we obtain:

$$
\partial_{t} j_{t}(x)=\frac{1}{2}\left\{\left[D_{\varepsilon}, j_{t}(x)\right] b_{t}^{\varepsilon}+b_{t}^{\varepsilon}\left[D_{\varepsilon}, j_{t}(x)\right]+\left[b_{t}^{\varepsilon}, j_{t}(x)\right] D_{\varepsilon}+D_{\varepsilon}\left[b_{t}^{\varepsilon}, j_{t}(x)\right]\right\} .
$$

Now denote

$$
x_{t}:=j_{t}(x) \text {, }
$$

and notice that

$$
\left\{D_{\varepsilon},\left[b_{t}^{\varepsilon}, x_{t}\right]\right\}=\left[b_{t}^{\varepsilon},\left\{D_{\varepsilon}, x_{t}\right\}\right]
$$

Then

$$
\partial_{t} x_{t}=\left[\frac{1}{2} D_{\varepsilon}, x_{t}\right] b_{t}^{\varepsilon}+b_{t}^{\varepsilon}\left[\frac{1}{2} D_{\varepsilon}, x_{t}\right]+\left[b_{t}^{\varepsilon},\left\{D_{\varepsilon}, x_{t}\right\}\right] .
$$

Therefore, with the notation

$$
\delta_{\varepsilon}(z):=\left[\frac{1}{2} D_{\varepsilon}, z\right], \quad \alpha_{\varepsilon}(z)=\left\{D_{\varepsilon}, z\right\},
$$

we see that the $\delta_{\varepsilon}$, are derivations and the equation satisfied by $x_{t}$ becomes

$$
\partial_{t} x_{t}=\delta_{\varepsilon}\left(x_{t}\right) b_{t}^{\varepsilon}+b_{t}^{\varepsilon} \delta_{\varepsilon}\left(x_{t}\right)+\left[b_{t}^{\varepsilon}, \alpha_{\varepsilon}\left(x_{t}\right)\right],
$$

equivalently

$$
\partial_{t} x_{t}=\left\{b_{t}^{\varepsilon}, \delta_{\varepsilon}\left(x_{t}\right)\right\}+\left[b_{t}^{\varepsilon}, \alpha_{\varepsilon}\left(x_{t}\right)\right]=\delta_{t}\left(x_{t}\right) .
$$

Notice that both $\alpha_{\varepsilon}$ and $\delta_{\varepsilon}$ act on the system algebra. 


\section{Normally Ordered Backward Heisenberg Evolutions}

Definition 3.1. A bounded white noise derivation is a set of bounded linear maps $\left\{\delta_{\varepsilon}, \alpha_{\varepsilon}\right\}(\varepsilon \in\{ \pm 1,0,2\})$ on $\mathcal{A}$, both of system type and such that each $\delta_{\varepsilon}$ is a *-derivation on $\mathcal{B}_{S}$ and:

$$
\left\{b_{t}^{\varepsilon}, \delta_{\varepsilon}(\cdot)\right\}+\left[b_{t}^{\varepsilon}, \alpha_{\varepsilon}(\cdot)\right]=: i \delta_{I, t}
$$

is a $*$-derivation on $\mathcal{B}_{S} \otimes \mathcal{B}_{R}$.

Lemma 3.1. The maps $\left\{\delta_{\varepsilon}, \alpha_{\varepsilon}\right\}(\varepsilon \in\{ \pm 1,0,2\})$ are a white noise derivation if and only if, for any $x, y \in \mathcal{A}$ and any $\varepsilon \in\{ \pm 1,0,2\}$,

$$
\left[b_{t}^{\varepsilon}, \alpha_{\varepsilon}(x y)\right]-\left[b_{t}^{\varepsilon}, \alpha_{\varepsilon}(x)\right] y-x\left[b_{t}^{\varepsilon}, \alpha_{\varepsilon}(y)\right]=\delta_{\varepsilon}(x)\left[b_{t}^{\varepsilon}, y\right]+\left[x, b_{t}^{\varepsilon}\right] \delta_{\varepsilon}(y) .
$$

Proof. The derivation property of $\delta_{I, t}$ gives:

$$
\begin{aligned}
\delta_{I, t}(x y) & =\delta_{I, t}(x) y+x \delta_{I, t}(y) \\
& =\left\{b_{t}^{\varepsilon}, \delta_{\varepsilon}(x y)\right\}+\left[b_{t}^{\varepsilon}, \alpha_{\varepsilon}(x y)\right] .
\end{aligned}
$$

Using (2.29) and the derivation property of $\delta_{\varepsilon}$

$$
\begin{aligned}
& \left\{b_{t}^{\varepsilon}, \delta_{\varepsilon}(x) y\right\}+\left\{b_{t}^{\varepsilon}, x \delta_{\varepsilon}(y)\right\}+\left[b_{t}^{\varepsilon}, \alpha_{\varepsilon}(x y)\right] \\
& \quad=\left\{b_{t}^{\varepsilon}, \delta_{\varepsilon}(x)\right\} y+\delta_{\varepsilon}(x)\left[y, b_{t}^{\varepsilon}\right]+x\left\{b_{t}^{\varepsilon}, \delta_{\varepsilon}(y)\right\}+\left[b_{t}^{\varepsilon}, x\right] \delta_{\varepsilon}(y)+\left[b_{t}^{\varepsilon}, \alpha_{\varepsilon}(x y)\right] .
\end{aligned}
$$

On the other hand,

$$
\delta_{I, t}(x) y+x \delta_{I, t}(y)=\left[\left\{b_{t}^{\varepsilon}, \delta_{\varepsilon}(x)\right\}+\left[b_{t}^{\varepsilon}, \alpha_{\varepsilon}(x)\right]\right] y+x\left[\left\{b_{t}^{\varepsilon}, \delta_{\varepsilon}(y)\right\}+\left[b_{t}^{\varepsilon}, \alpha_{\varepsilon}(y)\right]\right] .
$$

This is equivalent to

$$
\delta_{\varepsilon}(x)\left[y, b_{t}^{\varepsilon}\right]+\left[b_{t}^{\varepsilon}, x\right] \delta_{\varepsilon}(y)+\left[b_{t}^{\varepsilon}, \alpha_{\varepsilon}(x y)\right]=\left[b_{t}^{\varepsilon}, \alpha_{\varepsilon}(x)\right] y+x\left[b_{t}^{\varepsilon}, \alpha_{\varepsilon}(y)\right],
$$

and this is equivalent to (3.1).

Theorem 3.1. In the notations of Definition 3.1, assume that the map $1-\gamma_{-}\left(\delta_{2}+\right.$ $\left.\alpha_{2}\right)$ is invertible and define

$$
\begin{aligned}
& \tau_{+}=\left(1-\gamma_{-}\left(\delta_{2}+\alpha_{2}\right)\right)^{-1}, \\
& \tau_{-}=1+\gamma_{-}\left(\delta_{2}-\alpha_{2}\right) .
\end{aligned}
$$

Then the normally ordered form of the white noise backward Heisenberg equation:

$$
\partial_{t} j_{t}(x)=\delta_{I, t}\left(j_{t}(x)\right)=\left\{b_{t}^{\varepsilon}, \delta_{\varepsilon}\left(j_{t}(x)\right)\right\}+\left[b_{t}^{\varepsilon}, \alpha_{\varepsilon}\left(j_{t}(x)\right)\right]
$$

is:

$$
\begin{aligned}
& \partial_{t} j_{t}(x) \\
& \quad=b_{t}^{+}\left[\delta_{1}\left\{\left(1+\tau_{+}^{*} \tau_{-}^{*}\right) j_{t}(x)\right\}+\alpha_{1}\left\{\left(1-\tau_{+}^{*} \tau_{-}^{*}\right) j_{t}(x)\right\}+2 \gamma_{-}\left(\delta_{2}+\alpha_{2}\right)\left(\tau_{+} \delta_{1}\left(j_{t}(x)\right)\right)\right]
\end{aligned}
$$




$$
\begin{aligned}
& +\left[\delta_{-1}\left\{\left(\tau_{+} \tau_{-}+1\right) j_{t}(x)\right\}+\alpha_{-1}\left\{\left(\tau_{+} \tau_{-} 1\right) j_{t}(x)\right\}+2 \bar{\gamma}_{-}\left(\delta_{2}-\alpha_{2}\right)\left(\tau_{+}^{*} \delta_{1}^{*}\left(j_{t}(x)\right)\right)\right] b_{t} \\
& +b_{t}^{+}\left[\left(\delta_{2}+\alpha_{2}\right)\left(\tau_{+} \tau_{-} j_{t}(x)\right)+\left(\delta_{2}-\alpha_{2}\right)\left(\tau_{+}^{*} \tau_{-}^{*} j_{t}(x)\right)\right] b_{t} \\
& +2 \gamma_{-}\left(\delta_{-1}+\alpha_{-1}\right)\left(\tau_{+} \delta_{1}\left(j_{t}(x)\right)\right)+2 \bar{\gamma}_{-}\left(\delta_{1}-\alpha_{1}\right)\left(\tau_{+}^{*} \delta_{1}^{*}\left(j_{t}(x)\right)\right)+2 \delta_{0}\left(j_{t}(x)\right) .
\end{aligned}
$$

Proof. Equation (3.6) is equivalent to

$$
\begin{aligned}
j_{t}(x)= & x+\int_{0}^{t} d s \delta_{I, s}\left(j_{s}(x)\right) \\
= & x+\int_{0}^{t} d s\left\{b_{s}^{\varepsilon}, \delta_{\varepsilon}\left(j_{s}(x)\right)\right\}+\left[b_{s}^{\varepsilon}, \alpha_{\varepsilon}\left(j_{s}(x)\right)\right] \\
= & x+\int_{0}^{t} d s\left(\left\{b_{s}^{+}, \delta_{1}\left(j_{s}(x)\right)\right\}+\left[b_{s}^{+}, \alpha_{1}\left(j_{s}(x)\right)\right]+\left\{b_{s}, \delta_{-1}\left(j_{s}(x)\right)\right\}\right. \\
& +\left[b_{s}, \alpha_{-1}\left(j_{s}(x)\right)\right]+\left\{b_{s}^{+} b_{s}, \delta_{2}\left(j_{s}(x)\right)\right\}+\left[b_{s}^{+} b_{s}, \alpha_{2}\left(j_{s}(x)\right)\right] \\
& \left.+\left\{1, \delta_{0}\left(j_{s}(x)\right)\right\}+\left[1, \alpha_{0}\left(j_{s}(x)\right)\right]\right) .
\end{aligned}
$$

To put the last equation in normally ordered form, we use the time consecutive principle and the relation $\left[b_{t}, \delta_{\varepsilon}\left(j_{s}(x)\right)\right]=\delta_{\varepsilon}\left(\left[b_{t}, j_{s}(x)\right]\right)$, then by applying same technic as Corollary 2.2, we get:

$$
\left(1-\gamma_{-}\left(\delta_{2}+\alpha_{2}\right)\right) b_{t} j_{t}(x)=2 \gamma_{-} \delta_{1}\left(j_{t}(x)\right)+\left(1+\gamma_{-}\left(\delta_{2}-\alpha_{2}\right)\right) j_{t}(x) b_{t} .
$$

From the definition of (3.4), (3.5) we obtain

$$
b_{t} j_{t}(x)=2 \gamma_{-} \tau_{+} \delta_{1}\left(j_{t}(x)\right)+\tau_{+} \tau_{-} j_{t}(x) b_{t} .
$$

Then

$$
j_{t}(x) b_{t}^{+}=2 \bar{\gamma}_{-} \tau_{+}^{*} \delta_{1}^{*}\left(j_{t}(x)\right)+\tau_{+}^{*} \tau_{-}^{*} b_{t}^{+} j_{t}(x) .
$$

Now, let us use (3.9) and (3.10) to put (3.6) in normally ordered form:

$$
\begin{aligned}
\partial_{t} j_{t}(x)= & \delta_{1}\left(b_{t}^{+} j_{t}(x)\right)+\delta_{1}\left(j_{t}(x) b_{t}^{+}\right)+\alpha_{1}\left(b_{t}^{+} j_{t}(x)\right)-\alpha_{1}\left(j_{t}(x) b_{t}^{+}\right) \\
+ & \delta_{-1}\left(b_{t} j_{t}(x)\right)+\delta_{-1}\left(j_{t}(x) b_{t}\right)+\alpha_{-1}\left(b_{t} j_{t}(x)\right)-\alpha_{-1}\left(j_{t}(x) b_{t}\right) \\
+ & \delta_{2}\left(b_{t}^{+} b_{t} j_{t}(x)\right)+\delta_{2}\left(j_{t}(x) b_{t}^{+} b_{t}\right)+\alpha_{2}\left(b_{t}^{+} b_{t} j_{t}(x)\right) \\
- & \alpha_{2}\left(j_{t}(x) b_{t}^{+} b_{t}\right)+2 \delta_{0}\left(j_{t}(x)\right) \\
\partial_{t} j_{t}(x)= & \delta_{1}\left(b_{t}^{+} j_{t}(x)\right)+\delta_{1}\left(2 \bar{\gamma}_{-} \tau_{+}^{*} \delta_{1}^{*}\left(j_{t}(x)\right)+\tau_{+}^{*} \tau_{-}^{*} b_{t}^{+} j_{t}(x)\right) \\
& +\alpha_{1}\left(b_{t}^{+} j_{t}(x)\right)-\alpha_{1}\left(2 \bar{\gamma}_{-} \tau_{+}^{*} \delta_{1}^{*}\left(j_{t}(x)\right)+\tau_{+}^{*} \tau_{-}^{*} b_{t}^{+} j_{t}(x)\right) \\
& +\delta_{-1}\left(2 \gamma_{-} \tau_{+} \delta_{1}\left(j_{t}(x)\right)+\tau_{+} \tau_{-} j_{t}(x) b_{t}\right)+\delta_{-1}\left(j_{t}(x) b_{t}\right) \\
& +\alpha_{-1}\left(2 \gamma_{-} \tau_{+} \delta_{1}\left(j_{t}(x)\right)+\tau_{+} \tau_{-} j_{t}(x) b_{t}\right)-\alpha_{-1}\left(j_{t}(x) b_{t}\right)
\end{aligned}
$$




$$
\begin{aligned}
& +\delta_{2}\left(b_{t}^{+}\left[2 \gamma_{-} \tau_{+} \delta_{1}\left(j_{t}(x)\right)+\tau_{+} \tau_{-} j_{t}(x) b_{t}\right]\right) \\
& +\delta_{2}\left(\left[2 \bar{\gamma}_{-} \tau_{+}^{*} \delta_{1}^{*}\left(j_{t}(x)\right)+\tau_{+}^{*} \tau_{-}^{*} b_{t}^{+} j_{t}(x)\right] b_{t}\right) \\
& +\alpha_{2}\left(b_{t}^{+}\left[2 \gamma_{-} \tau_{+} \delta_{1}\left(j_{t}(x)\right)+\tau_{+} \tau_{-} j_{t}(x) b_{t}\right]\right) \\
& -\alpha_{2}\left(\left[2 \bar{\gamma}_{-} \tau_{+}^{*} \delta_{1}^{*}\left(j_{t}(x)\right)+\tau_{+}^{*} \tau_{-}^{*} b_{t}^{+} j_{t}(x)\right] b_{t}\right)+2 \delta_{0}\left(j_{t}(x)\right),
\end{aligned}
$$

this leads to

$$
\begin{aligned}
\partial_{t} j_{t}(x)= & b_{t}^{+} \delta_{1}\left(j_{t}(x)\right)+2 \bar{\gamma}_{-} \delta_{1}\left(\tau_{+}^{*} \delta_{1}^{*}\left(j_{t}(x)\right)\right)+b_{t}^{+} \delta_{1}\left(\tau_{+}^{*} \tau_{-}^{*} j_{t}(x)\right) \\
& +b_{t}^{+} \alpha_{1}\left(j_{t}(x)\right)-2 \bar{\gamma}_{-} \alpha_{1}\left(\tau_{+}^{*} \delta_{1}^{*}\left(j_{t}(x)\right)\right)+b_{t}^{+} \alpha_{1}\left(\tau_{+}^{*} \tau_{-}^{*} j_{t}(x)\right) \\
& +2 \gamma_{-} \delta_{-1}\left(\tau_{+} \delta_{1}\left(j_{t}(x)\right)\right)+\delta_{-1}\left(\tau_{+} \tau_{-} j_{t}(x)\right) b_{t}+\delta_{-1}\left(j_{t}(x)\right) b_{t} \\
& +2 \gamma_{-} \alpha_{-1}\left(\tau_{+} \delta_{1}\left(j_{t}(x)\right)\right)+\alpha_{-1}\left(\tau_{+} \tau_{-} j_{t}(x)\right) b_{t}-\alpha_{-1}\left(j_{t}(x)\right) b_{t} \\
& +2 \gamma_{-} b_{t}^{+} \delta_{2}\left(\tau_{+} \delta_{1}\left(j_{t}(x)\right)\right)+b_{t}^{+} \delta_{2}\left(\tau_{+} \tau_{-} j_{t}(x)\right) b_{t}+2 \bar{\gamma}_{-} \delta_{2}\left(\tau_{+}^{*} \delta_{1}^{*}\left(j_{t}(x)\right)\right) b_{t} \\
& +b_{t}^{+} \delta_{2}\left(\tau_{+}^{*} \tau_{-}^{*} j_{t}(x)\right) b_{t}+2 \gamma_{-} b_{t}^{+} \alpha_{2}\left(\tau_{+} \delta_{1}\left(j_{t}(x)\right)\right)+b_{t}^{+} \alpha_{2}\left(\tau_{+} \tau_{-} j_{t}(x)\right) b_{t} \\
& -2 \bar{\gamma}_{-} \alpha_{2}\left(\tau_{+}^{*} \delta_{1}^{*}\left(j_{t}(x)\right)\right) b_{t}-b_{t}^{+} \alpha_{2}\left(\tau_{+}^{*} \tau_{-}^{*} j_{t}(x)\right) b_{t}+2 \delta_{0}\left(j_{t}(x)\right)
\end{aligned}
$$

Then, the normally ordered form of (3.6) is equivalent to Eq. (3.7).

\section{Homomorphic White Noise Backward Heisenberg Evolutions}

Theorem 4.1. Let $\delta_{2}, \delta_{1}, \delta_{-1}$ and $\delta_{0}$ be bounded linear maps acting on the bounded operators on the initial space and let us denote with the same symbols the linear extensions of these operators to $\mathcal{B}\left(\mathcal{H}_{S} \otimes \Gamma\right) \equiv \mathcal{B}\left(\mathcal{H}_{S}\right) \otimes \mathcal{B}(\Gamma)$ characterized by:

$b_{S} \otimes b_{\Gamma} \in \mathcal{B}\left(\mathcal{H}_{S}\right) \otimes \mathcal{B}(\Gamma) \mapsto \delta_{\varepsilon}\left(b_{S}\right) \otimes b_{\Gamma} \in \mathcal{B}\left(\mathcal{H}_{S}\right) \otimes \mathcal{B}(\Gamma) ; \quad \varepsilon \in\{ \pm 1,0,2\}$.

The unique solution of the backward flow equation

$$
\begin{gathered}
\partial_{t} j_{t}(x)=b_{t}^{+} \delta_{2}\left(j_{t}(x)\right) b_{t}+b_{t}^{+} \delta_{1}\left(j_{t}(x)\right)+\delta_{-1}\left(j_{t}(x)\right) b_{t}+\delta_{0}\left(j_{t}(x)\right) ; \\
j_{0}(x)=x \quad \forall x \in B\left(\mathcal{H}_{S}\right),
\end{gathered}
$$

is an identity preserving $*$-homomorphism if and only if the maps $\delta_{\varepsilon}(\varepsilon \in\{ \pm 1,0,2\})$ satisfy the following conditions:

$$
\begin{gathered}
\delta_{2}(1)=\delta_{1}(1)=\delta_{0}(1)=\delta_{-1}(1)=0, \\
\delta_{-1}(x)=\delta_{1}\left(x^{*}\right)^{*}, \\
\delta_{1}(x)=\delta_{-1}\left(x^{*}\right)^{*}, \\
\delta_{0}(x)=\delta_{0}\left(x^{*}\right)^{*} \\
\delta_{2}(x)=\delta_{2}\left(x^{*}\right)^{*}
\end{gathered}
$$




$$
\begin{aligned}
\delta_{2}(x y) & =\delta_{2}(x) y+x \delta_{2}(y)+2 \operatorname{Re} \gamma_{-} \delta_{2}(x) \delta_{2}(y), \\
\delta_{1}(x y) & =\delta_{1}(x) y+x \delta_{1}(y)+2 \operatorname{Re} \gamma_{-} \delta_{2}(x) \delta_{1}(y), \\
\delta_{-1}(x y) & =\delta_{-1}(x) y+x \delta_{-1}(y)+2 \operatorname{Re} \gamma_{-} \delta_{-1}(x) \delta_{2}(y), \\
\delta_{0}(x y) & =\delta_{0}(x) y+x \delta_{0}(y)+2 \operatorname{Re} \gamma_{-} \delta_{-1}(x) \delta_{1}(y) .
\end{aligned}
$$

Proof. The condition $j_{t}(1)=1$, is equivalent to $\partial_{t} j_{t}(1)=0$ and leads to:

$$
b_{t}^{+} \delta_{2}\left(j_{t}(1)\right) b_{t}+b_{t}^{+} \delta_{1}\left(j_{t}(1)\right)+\delta_{-1}\left(j_{t}(1)\right) b_{t}+\delta_{0}\left(j_{t}(1)\right)=0 .
$$

The independence of the basic noises then implies that

$$
\delta_{2}(1)=\delta_{1}(1)=\delta_{0}(1)=\delta_{-1}(1)=0
$$

and, evaluating this at $t=0$, we find (4.2). Replacing $x$ by $x^{*}(5.1)$ becomes:

$$
\partial_{t} j_{t}\left(x^{*}\right)=b_{t}^{+} \delta_{2}\left(j_{t}\left(x^{*}\right)\right) b_{t}+b_{t}^{+} \delta_{1}\left(j_{t}\left(x^{*}\right)\right)+\delta_{-1}\left(j_{t}\left(x^{*}\right)\right) b_{t}+\delta_{0}\left(j_{t}\left(x^{*}\right)\right) .
$$

On the other hand, by the $*$-homomorphism condition, this must be equal to:

$$
\partial_{t} j_{t}(x)^{*}=b_{t}^{+} \delta_{2}\left(j_{t}(x)\right)^{*} b_{t}+\delta_{1}\left(j_{t}(x)\right)^{*} b_{t}+b_{t}^{+} \delta_{-1}\left(j_{t}(x)\right)^{*}+\delta_{0}\left(j_{t}(x)\right)^{*} .
$$

By the same argument as above we obtain the conditions (4.3)-(4.6).

To exploit the homomorphism condition we calculate the causal commutators $\left[b_{t}, j_{t}(x)\right]$ and $\left[b_{t}, j_{t}\left(x^{*}\right)\right]$. The former gives:

$$
\left[b_{t}, j_{t}(x)\right]=\gamma_{-} \delta_{2}\left(j_{t}(x)\right) b_{t}+\gamma_{-} \delta_{1}\left(j_{t}(x)\right),
$$

or equivalently:

$$
b_{t} j_{t}(x)=\left(1+\gamma_{-} \delta_{2}\right)\left(j_{t}(x)\right) b_{t}+\gamma_{-} \delta_{1}\left(j_{t}(x)\right)=\rho\left(j_{t}(x)\right) b_{t}+\gamma_{-} \delta_{1}\left(j_{t}(x)\right) ;
$$

where we denote

$$
\rho:=\left(1+\gamma_{-} \delta_{2}\right)
$$

Similarly, one has:

$$
\left[b_{t}, j_{t}(x)^{*}\right]=\gamma_{-} \delta_{2}\left(j_{t}(x)\right)^{*} b_{t}+\gamma_{-} \delta_{-1}\left(j_{t}(x)\right)^{*},
$$

and, taking adjoint:

$$
j_{t}(x) b_{t}^{+}=b_{t}^{+}\left(1+\bar{\gamma}_{-} \delta_{2}\right)\left(j_{t}(x)\right)+\bar{\gamma}_{-} \delta_{-1}\left(j_{t}(x)\right)=b_{t}^{+} \rho^{\prime}\left(j_{t}(x)\right)+\bar{\gamma}_{-} \delta_{-1}\left(j_{t}(x)\right) ;
$$

where we denote

$$
\rho^{\prime}=\left(1+\bar{\gamma}_{-} \delta_{2}\right)
$$

Equation (5.1), applied to $x y$ gives:

$$
\partial_{t} j_{t}(x y)=b_{t}^{+} \delta_{2}\left(j_{t}(x y)\right) b_{t}+b_{t}^{+} \delta_{1}\left(j_{t}(x y)\right)+\delta_{-1}\left(j_{t}(x y)\right) b_{t}+\delta_{0}\left(j_{t}(x y)\right),
$$


but, from $j_{t}(x y)=j_{t}(x) j_{t}(y)$ and the Leibnitz rule for white noise derivatives, we deduce also:

$$
\begin{aligned}
\partial_{t} j_{t}(x y)= & \partial_{t} j_{t}(x) j_{t}(y)+j_{t}(x) \partial_{t} j_{t}(y) \\
= & \left\{b_{t}^{+} \delta_{2}\left(j_{t}(x)\right) b_{t}+b_{t}^{+} \delta_{1}\left(j_{t}(x)\right)+\delta_{-1}\left(j_{t}(x)\right) b_{t}+\delta_{0}\left(j_{t}(x)\right)\right\} j_{t}(y) \\
& +j_{t}(x)\left\{b_{t}^{+} \delta_{2}\left(j_{t}(y)\right) b_{t}+b_{t}^{+} \delta_{1}\left(j_{t}(y)\right)+\delta_{-1}\left(j_{t}(y)\right) b_{t}+\delta_{0}\left(j_{t}(y)\right)\right\} \\
= & b_{t}^{+} \delta_{2}\left(j_{t}(x)\right)\left\{\rho\left(j_{t}(y)\right) b_{t}+\gamma_{-} \delta_{1}\left(j_{t}(y)\right)\right\}+b_{t}^{+} \delta_{1}\left(j_{t}(x)\right) j_{t}(y) \\
& +\delta_{-1}\left(j_{t}(x)\right)\left\{\rho\left(j_{t}(y)\right) b_{t}+\gamma_{-} \delta_{1}\left(j_{t}(y)\right)\right\}+\delta_{0}\left(j_{t}(x)\right) j_{t}(y) \\
& +\left\{b_{t}^{+} \rho^{\prime}\left(j_{t}(x)\right)+\bar{\gamma}_{-} \delta_{-1}\left(j_{t}(x)\right)\right\} \delta_{2}\left(j_{t}(y)\right) b_{t} \\
& +\left\{b_{t}^{+} \rho^{\prime}\left(j_{t}(x)\right)+\bar{\gamma}_{-} \delta_{-1}\left(j_{t}(x)\right)\right\} \delta_{1}\left(j_{t}(y)\right) \\
& +j_{t}(x) \delta_{-1}\left(j_{t}(y)\right) b_{t}+j_{t}(x) \delta_{0}\left(j_{t}(y)\right) \\
= & b_{t}^{+}\left\{\delta_{2}\left(j_{t}(x)\right) \rho\left(j_{t}(y)\right)+\rho^{\prime}\left(j_{t}(x)\right) \delta_{2}\left(j_{t}(y)\right)\right\} b_{t} \\
& +b_{t}^{+}\left\{\gamma_{-} \delta_{2}\left(j_{t}(x)\right) \delta_{1}\left(j_{t}(y)\right)+\delta_{1}\left(j_{t}(x)\right) j_{t}(y)+\rho^{\prime}\left(j_{t}(x)\right) \delta_{1}\left(j_{t}(y)\right)\right\} \\
& +\left\{\delta_{-1}\left(j_{t}(x)\right) \rho\left(j_{t}(y)\right)+\bar{\gamma}_{-} \delta_{-1}\left(j_{t}(x)\right) \delta_{2}\left(j_{t}(y)\right)+j_{t}(x) \delta_{-1}\left(j_{t}(y)\right)\right\} b_{t} \\
& +\left\{\gamma_{-} \delta_{-1}\left(j_{t}(x)\right) \delta_{1}\left(j_{t}(y)\right)+\delta_{0}\left(j_{t}(x)\right) j_{t}(y)\right. \\
& \left.+\bar{\gamma}_{-} \delta_{-1}\left(j_{t}(x)\right) \delta_{1}\left(j_{t}(y)\right)+j_{t}(x) \delta_{0}\left(j_{t}(y)\right)\right\} .
\end{aligned}
$$

Using the independence of the basic noises as before, one then gets:

$$
\begin{aligned}
\delta_{2}(x y) & =\delta_{2}(x) \rho(y)+\rho^{\prime}(x) \delta_{2}(y) \\
\delta_{1}(x y) & =\gamma_{-} \delta_{2}(x) \delta_{1}(y)+\delta_{1}(x) y+\rho^{\prime}(x) \delta_{1}(y) \\
\delta_{-1}(x y) & =\delta_{-1}(x) \rho(y)+\bar{\gamma}_{-} \delta_{-1}(x) \delta_{2}(y)+x \delta_{-1}(y) \\
\delta_{0}(x y) & =\gamma_{-} \delta_{-1}(x) \delta_{1}(y)+\delta_{0}(x) y+\bar{\gamma}_{-} \delta_{-1}(x) \delta_{1}(y)+x \delta_{0}(y) .
\end{aligned}
$$

Eventually, replacing $\rho$ and $\rho^{\prime}$ by their expressions, we obtain (4.7)-(4.10).

\section{White Noise Evans-Hudson Flows}

The following theorem is a white noise extension of the the Evans-Hudson structure equations which are recovered when $\operatorname{Re} \gamma_{-}=1 / 2$.

Theorem 5.1. Let $\delta_{2}, \delta_{1}, \delta_{-1}$ and $\delta_{0}$ be norm bounded linear maps acting on the algebra of all bounded operators on the initial space. Then the unique solution of the normally ordered forward flow equation:

$$
\partial_{t} j_{t}(x)=b_{t}^{+} j_{t}\left(\delta_{2}(x)\right) b_{t}+b_{t}^{+} j_{t}\left(\delta_{1}(x)\right)+j_{t}\left(\delta_{-1}(x)\right) b_{t}+j_{t}\left(\delta_{0}(x)\right),
$$

and

$$
j_{0}(x)=x \quad \forall x \in B\left(\mathcal{H}_{S}\right),
$$


is an identity preserving *-homomorphism of $\mathcal{B}\left(\mathcal{H}_{S}\right)$ if and only if the maps $\delta_{\varepsilon}$ $(\varepsilon \in\{ \pm 1,0,2\})$ satisfy the same conditions as Theorem 4.1 .

Proof. The steps of the proof are similar to those of the proof of Theorem 4.1.

\section{Inner White Noise Heisenberg Equations}

Corollary 6.1. The causally normally ordered form of the equation

$$
\partial_{t} U_{t}=-i\left(A b_{t}+B b_{t}^{+}+b_{t}^{+} T b_{t}+C\right) U_{t} ; \quad U_{0}=1,
$$

is, in the notation (2.20)

$$
\partial_{t} U_{t}=-i b_{t}^{+} K B U_{t}-i A K U_{t} b_{t}-i T K b_{t}^{+} U_{t} b_{t}+\left(-\gamma_{-} A K B-i C\right) U_{t} .
$$

Proof. Equation (2.2) can be written:

$$
\begin{aligned}
\partial_{t} U_{t} & =-i A b_{t} U_{t}-i B b_{t}^{+} U_{t}-i b_{t}^{+} T b_{t} U_{t}-i C U_{t} \\
& =-i B b_{t}^{+} U_{t}-i A\left[b_{t}, U_{t}\right]-i A U_{t} b_{t}-i b_{t}^{+} T\left[b_{t}, U_{t}\right]-i b_{t}^{+} T U_{t} b_{t}-i C U_{t}
\end{aligned}
$$

Therefore, from Corollary 2.2, we obtain:

$\partial_{t} U_{t}=-i B b_{t}^{+} U_{t}-i A\left(-i \gamma_{-} K B U_{t}+K U_{t} b_{t}\right)-i b_{t}^{+} T\left(-i \gamma_{-} K B U_{t}+K U_{t} b_{t}\right)-i C U_{t}$, equivalently,

$$
\partial_{t} U_{t}=b_{t}^{+}\left(-i B-\gamma_{-} T K B\right) U_{T}-i A K U_{t} b_{t}-i T K b_{t}^{+} U_{t} b_{t}+\left(-\gamma_{-} A K B-i C\right) U_{t} .
$$

Therefore (6.3) becomes (6.2).

\subsection{The forward inner Langevin equation}

Proposition 6.1. Let $U_{t}$ be the unique solution of Eq. (2.2). Define, for any bounded operator $x$ on the initial space $\mathcal{H}_{S}$ and for any $t \in \mathbb{R}_{+}$:

$$
j_{t}(x):=U_{t}^{*} x U_{t} .
$$

The equation satisfied by $j_{t}(x)$ is called the forward inner Langevin equation. Its causally normally ordered form is:

$$
\begin{aligned}
\partial_{t} j_{t}(x)= & b_{t}^{+} j_{t}\left(i K^{*} A^{*} x+\gamma_{-} K^{*} T^{*} x K B-i K^{*} x B-\gamma_{-} K^{*} x T K B\right) \\
& +j_{t}\left(i B^{*} x K-\bar{\gamma}_{-} B^{*} K^{*} T^{*} x K-i x A K+\bar{\gamma}_{-} B^{*} K^{*} x T K\right) b_{t} \\
& +b_{t}^{+} j_{t}\left(i K^{*} T^{*} x K-i K^{*} x T K\right) b_{t}+j_{t}\left(\gamma_{-} B^{*} x K B-\bar{\gamma}_{-} B^{*} K^{*} A^{*} x\right. \\
& +i\left|\gamma_{-}\right|^{2} B^{*} K^{*} T^{*} x K B+i C^{*} x+\bar{\gamma}_{-} B^{*} K^{*} x B-\gamma_{-} x A K B \\
& \left.-i\left|\gamma_{-}\right|^{2} B^{*} K^{*} x T K B-i x C\right)
\end{aligned}
$$

where $K$ is given by (2.20). 
Proof. The white noise equation satisfied by $j_{t}(x)$ is:

$$
\begin{aligned}
\partial_{t} j_{t}(x)= & \partial_{t}\left(U_{t}^{*} x U_{t}\right) \\
= & U_{t}^{*}\left(i B^{*} b_{t}+i b_{t}^{+} A^{*}+i b_{t}^{+} T^{*} b_{t}+i C^{*}\right) x U_{t} \\
& +U_{t}^{*} x\left(-i B b_{t}^{+}-i A b_{t}-i b_{t}^{+} T b_{t}-i C\right) U_{t} .
\end{aligned}
$$

From Corollary 2.2 one deduces that:

$$
\begin{aligned}
\partial_{t} j_{t}(x)= & U_{t}^{*}\left(i B^{*} x\right)\left(-i \gamma_{-} K B U_{t}+K U_{t} b_{t}\right)+\left(i \bar{\gamma}_{-} U_{t}^{*} B^{*} K^{*}+b_{t}^{+} U_{t}^{*} K^{*}\right)\left(i A^{*} x\right) U_{t} \\
& +\left(b_{t}^{+} U_{t}^{*} K^{*}+i \bar{\gamma}_{-} U_{t}^{*} B^{*} K^{*}\right)\left(i T^{*} x\right)\left(-i \gamma_{-} K B U_{t}+K U_{t} b_{t}\right)+U_{t}^{*}\left(i C^{*} x\right) U_{t} \\
& +\left(i \bar{\gamma}_{-} U_{t}^{*} B^{*} K^{*}+b_{t}^{+} U_{t}^{*} K^{*}\right)(-i x B) U_{t}+U_{t}^{*}(-i A x)\left(-i \gamma_{-} K B U_{t}+K U_{t} b_{t}\right) \\
& +\left(i \bar{\gamma}_{-} U_{t}^{*} B^{*} K^{*}+b_{t}^{+} U_{t}^{*} K^{*}\right)(-i T x)\left(-i \gamma_{-} K B U_{t}+K U_{t} b_{t}\right)+U_{t}^{*}(-i C x) U_{t} \\
= & j_{t}\left(\gamma_{-} B^{*} x K B\right)+j_{t}\left(i B^{*} x K\right) b_{t}+b_{t}^{+} j_{t}\left(i K^{*} A^{*} x\right)+j_{t}\left(-\bar{\gamma}_{-} B^{*} K^{*} A^{*} x\right) \\
& +b_{t}^{+} j_{t}\left(\gamma_{-} K^{*} T^{*} x K B\right)+b_{t}^{+} j_{t}\left(i K^{*} T^{*} x K\right) b_{t}+j_{t}\left(i\left|\gamma_{-}\right|^{2} B^{*} K^{*} T^{*} x K B\right) \\
& +j_{t}\left(-\bar{\gamma}_{-} B^{*} K^{*} T^{*} x K\right) b_{t}+j_{t}\left(i C^{*} x\right)+b_{t}^{+} j_{t}\left(-i K^{*} x B\right)+j_{t}\left(\bar{\gamma}_{-} B^{*} K^{*} x B\right) \\
& +j_{t}\left(-\gamma_{-} x A K B\right)+j_{t}(-i x A K) b_{t}+b_{t}^{+} j_{t}\left(-\gamma_{-} K^{*} x T K B\right) \\
& +b_{t}^{+} j_{t}\left(-i K^{*} x T K\right) b_{t}+j_{t}\left(-i\left|\gamma_{-}\right|^{2} B^{*} K^{*} x T K B\right) \\
& +j_{t}\left(\bar{\gamma}_{-} B^{*} K^{*} x T K\right) b_{t}+j_{t}(-i x C),
\end{aligned}
$$

and this is equivalent to $(6.5)$.

\subsection{White noise backward inner Heisenberg evolutions}

In the previous section we have discussed the normally ordered form of the equation satisfied by the forward flow $\left(U_{t}^{*} x U_{t}\right)$, associated to the white noise Hamiltonian equation (6.1). In this section we solve the same problem for the backward flow associated to the same equation.

Theorem 6.1. Consider the white noise Hamiltonian $H_{I}(t)$ given by:

$$
H_{I}(t)=D b_{t}^{+}+b_{t} D^{+}+T b_{t}^{+} b_{t}+C ;
$$

where $D, T$ and $C$ are elements of $B\left(\mathcal{H}_{S}\right)$ such that $T$ and $C$ are self-adjoint. Then the causally normally ordered form of the white noise Heisenberg equation:

$$
\partial_{t} j_{t}(x)=-i\left[H_{I}(t), j_{t}(x)\right],
$$

is:

$$
\begin{aligned}
\partial_{t} j_{t}(x)= & b_{t}^{+}\left(-i K D j_{t}(x)+i K^{*-1} j_{t}(x) K^{*} D+\gamma_{-} T K j_{t}(x) D\right) \\
& +\left(i j_{t}(x) D^{+} K^{*}-i D^{+} K j_{t}(x) K^{-1}+\bar{\gamma}_{-} D^{+} j_{t}(x) K^{*} T\right) b_{t} \\
& +b_{t}^{+}\left(-i T K j_{t}(x) K^{-1}+i K^{*-1} j_{t}(x) K^{*} T\right) b_{t} \\
& +\left(-\bar{\gamma}_{-}\left[j_{t}(x), D^{+}\right] K^{*} D-\gamma_{-} D^{+} K\left[D, j_{t}(x)\right]-i C j_{t}(x)+i j_{t}(x) C\right) .
\end{aligned}
$$


Proof. Consider the following white noise Hamiltonian equation:

$$
\partial_{t} j_{t}(x)=-i\left[H_{I}(t), j_{t}(x)\right] .
$$

We want to put in causal normal order the equation:

$$
\begin{aligned}
\partial_{t} j_{t}(x)= & -i\left(D b_{t}^{+} j_{t}(x)-j_{t}(x) D b_{t}^{+}+b_{t} D^{+} j_{t}(x)\right. \\
& \left.-j_{t}(x) D^{+} b_{t}+T b_{t}^{+} b_{t} j_{t}(x)-j_{t}(x) b_{t}^{+} b_{t} T+C j_{t}(x)-j_{t}(x) C\right) .
\end{aligned}
$$

To this goal we calculate the commutators $\left[b_{t}, j_{t}(x)\right]$ and $\left[b_{t}, j_{t}\left(x^{*}\right)\right]$ using the time consecutive principle. This gives:

$$
\left[b_{t}, j_{t}(x)\right]=-i \gamma_{-}\left[D, j_{t}(x)\right]-i \gamma_{-}\left(T b_{t} j_{t}(x)-j_{t}(x) b_{t} T\right) .
$$

In the notation $(2.20)$ (i.e. $\left.K=\left(1+i \gamma_{-} T\right)^{-1}\right)$ this is equivalent to

$$
b_{t} j_{t}(x)=-i \gamma_{-} K\left[D, j_{t}(x)\right]+K j_{t}(x) b_{t} K^{-1} .
$$

From the equation for $j_{t}\left(x^{*}\right)$ we deduce:

$$
b_{t} j_{t}\left(x^{*}\right)=i \gamma_{-} K\left[j_{t}\left(x^{*}\right), D\right]+K j_{t}\left(x^{*}\right) b_{t} K^{-1},
$$

it follows that:

$$
j_{t}(x) b_{t}^{+}=+i \bar{\gamma}_{-}\left[j_{t}(x), D^{+}\right] K^{*}+K^{*-1} b_{t}^{+} j_{t}(x) K^{*}
$$

Then

$$
\begin{aligned}
\partial_{t} j_{t}(x)= & -i\left(b_{t}^{+} D j_{t}(x)-\left\{i \bar{\gamma}_{-}\left[j_{t}(x), D^{+}\right] K^{*}+K^{*-1} b_{t}^{+} j_{t}(x) K^{*}\right\} D\right. \\
& +D^{+}\left\{-i \gamma_{-} K\left[D, j_{t}(x)\right]+K j_{t}(x) b_{t} K^{-1}\right\}-j_{t}(x) D^{+} b_{t} \\
& +T b_{t}^{+}\left\{-i \gamma_{-} K\left[D, j_{t}(x)\right]+K j_{t}(x) b_{t} K^{-1}\right\} \\
& \left.-\left\{i \bar{\gamma}_{-}\left[j_{t}(x), D^{+}\right] K^{*}+K^{*-1} b_{t}^{+} j_{t}(x) K^{*}\right\} b_{t} T-i C j_{t}(x)+i j_{t}(x) C\right) \\
= & b_{t}^{+}\left(-i D j_{t}(x)+i K^{*-1} j_{t}(x) K^{*} D-\gamma_{-} T K\left[D, j_{t}(x)\right]\right) \\
& +\left(i j_{t}(x) D^{+}-i D^{+} K j_{t}(x) K^{-1}-\bar{\gamma}_{-}\left[j_{t}(x), D^{+}\right] K^{*} T\right) b_{t} \\
& +b_{t}^{+}\left(-i T K j_{t}(x) K^{-1}+i K^{*-1} j_{t}(x) K^{*} T\right) b_{t} \\
& \left(-\bar{\gamma}_{-}\left[j_{t}(x), D^{+}\right] K * D-\gamma_{-} D^{+} K\left[D, j_{t}(x)\right]+C j_{t}(x)-j_{t}(x) C\right)
\end{aligned}
$$

and this is equivalent to (6.8).

The following is the analogue of Theorem 8.1 for backward flows.

\subsection{The structure maps in terms of the Hamiltonian coefficients}

Combining the results of Theorems 4.1 and 6.1 , we obtain the expression of the structure maps as functions of the original Hamiltonian coefficients. 
Corollary 6.2. In the notations of Theorem 6.1, the structure maps are given by:

$$
\begin{aligned}
\delta_{2}(x) & =-i T K x K^{-1}+i K^{*-1} x K^{*} T, \\
\delta_{1}(x) & =-i K D x+i K^{*-1} x K^{*} D+\gamma_{-} T K x D, \\
\delta_{-1}(x) & =i x D^{+} K^{*}-i D^{+} K x K^{-1}+\bar{\gamma}_{-} D^{+} x K^{*} T, \\
\delta_{0}(x) & =-\bar{\gamma}_{-}\left[x, D^{+}\right] K^{*} D-\gamma_{-} D^{+} K[D, x]+C x-x C .
\end{aligned}
$$

\subsection{The backward inner Langevin equation}

Proposition 6.2. The backward flow is defined, for any bounded operator $x$ on the initial space $\mathcal{H}_{S}$ and for any $t \in \mathbb{R}$ by:

$$
j_{t}(x)=U_{t} x U_{t}^{*} .
$$

The causally normally ordered form of the backward inner Langevin equation satisfied by $j_{t}(x)$ is:

$$
\begin{aligned}
\partial_{t} j_{t}(x)= & b_{t}^{+}\left(-i B-\gamma_{-} T K B\right) j_{t}(x)+\gamma_{-} b_{t}^{+} T K j_{t}(x) A^{*}+i K^{\prime *} b_{t}^{+} j_{t}(x) K^{*} A^{*} \\
& -i A K j_{t}(x) b_{t} K^{\prime}+j_{t}(x)\left(i B^{*}-\bar{\gamma}_{-} B^{*} K^{*} T^{*}\right) b_{t}+\bar{\gamma}_{-} A j_{t}(x) K^{*} T^{*} b_{t} \\
& -i b_{t}^{+} T K j_{t}(x) b_{t} K^{\prime}+i K^{\prime *} b_{t}^{+} j_{t}(x) K^{*} T^{*} b_{t} \\
& -i A\left(-i \gamma_{-} K B j_{t}(x)+i \gamma_{-} K j_{t}(x) A^{*}\right) \\
& -i C j_{t}(x)-\bar{\gamma}_{-} j_{t}(x) B^{*} K^{*} A^{*}+\bar{\gamma}_{-} A j_{t}(x) K^{*} A^{*}+i j_{t}(x) C^{*}
\end{aligned}
$$

Proof. Differentiating by the Leibnitz rule the product $U_{t} x U_{t}^{*}$ one finds:

$$
\begin{aligned}
\partial_{t} j_{t}(x)= & \partial_{t} U_{t} x U_{t}^{*}=\left(\partial_{t} U_{t}\right) x U_{t}^{*}+U_{t} x\left(\partial_{t} U_{t}^{*}\right) \\
= & \left(-i B b_{t}^{+}-i A b_{t}-i b_{t}^{+} T b_{t}-i C\right) U_{t} x U_{t}^{*} \\
& +U_{t} x U_{t}^{*}\left(i B^{*} b_{t}+i b_{t}^{+} A^{*}+i b_{t}^{+} T^{*} b_{t}+i C^{*}\right) .
\end{aligned}
$$

From Corollary 2.2, and in the same notations, we obtain:

$$
\begin{aligned}
b_{t} j_{t}(x) & =b_{t} U_{t} x U_{t}^{*}=\left(-i \gamma_{-} K B U_{t}+K U_{t} b_{t}\right) x U_{t}^{*} \\
& =-i \gamma_{-} K B j_{t}(x)+K U_{t} x\left(i \gamma_{-} U_{t}^{*} A^{*}+U_{t}^{*} b_{t} K^{\prime}\right) \\
& =-i \gamma_{-} K B j_{t}(x)+i \gamma_{-} K j_{t}(x) A^{*}+K j_{t}(x) b_{t} K^{\prime}
\end{aligned}
$$

Therefore:

$$
\begin{aligned}
\partial_{t} j_{t}(x) & =-i B b_{t}^{+} j_{t}(x)-i A\left(-i \gamma_{-} K B j_{t}(x)+i \gamma_{-} K j_{t}(x) A^{*}+K j_{t}(x) b_{t} K^{\prime}\right) \\
& =-i b_{t}^{+} T\left(-i \gamma_{-} K B j_{t}(x)+i \gamma_{-} K j_{t}(x) A^{*}+K j_{t}(x) b_{t} K^{\prime}\right) \\
& =-i C j_{t}(x)+i j_{t}(x) b_{t} B^{*}+i\left(i \bar{\gamma}_{-} j_{t}(x) B^{*} K^{*}-i \bar{\gamma}_{-} A j_{t}(x) K^{*}\right.
\end{aligned}
$$




$$
\begin{aligned}
& \left.+k^{\prime *} b_{t}^{+} j_{t}(x) K^{*}\right) A^{*} \\
= & +i\left(i \bar{\gamma}_{-} j_{t}(x) B^{*} K^{*}-i \bar{\gamma}_{-} A j_{t}(x) K^{*}+k^{\prime *} b_{t}^{+} j_{t}(x) K^{*}\right) T^{*} b_{t}+i j_{t}(x) C^{*} .
\end{aligned}
$$

By grouping together the homogeneous terms we find (6.11).

\section{The Unitarity Conditions}

\subsection{The isometricity condition}

The following theorem shows that the isometry condition on an adapted solution of the equation

$$
\partial_{t} U_{t}=-i\left(A b_{t}+B b_{t}^{+}+b_{t}^{+} T b_{t}+C\right) U_{t}, \quad U_{0}=1,
$$

is equivalent to the formal self-adjointness of the operator valued distribution:

$$
A b_{t}+B b_{t}^{+}+b_{t}^{+} T b_{t}+C .
$$

Theorem 7.1. Let $A, B, C$ and $T$ be bounded operators on the initial space $\mathcal{H}_{S}$ then, for the unique solution $U_{t}$ of $E q$. (7.1), the following statements are equivalent:

(i) $U_{t}$ is isometric, i.e.

$$
U_{t}^{*} U_{t}=1
$$

and both $\left(1+i \gamma_{-} T\right)$ and $\left(1+i \gamma_{-} T^{*}\right)$ are invertible.

(ii) The coefficients $A, B, T$ and $C$ satisfy the relations:

$$
\begin{gathered}
T=T^{*}, \\
B^{*}=A, \\
C=C^{*}
\end{gathered}
$$

so that Eq. (7.1) takes the form

$$
\partial_{t} U_{t}=-i\left(A b_{t}+A^{*} b_{t}^{+}+b_{t}^{+} T b_{t}+C\right) U_{t}, \quad U_{0}=1 .
$$

Proof. The isometry condition is equivalent to

$$
j_{t}(1)=1 \quad \forall t
$$

where $j_{t}$ is given by (6.4). Thus condition (7.6) is equivalent to the fact that the right-hand side of (6.5) vanishes for all $t$. The independence of the basic noises implies then that the coefficients of the various terms on the right-hand side of (6.5) vanish separately. The vanishing of the $b_{t}^{+} b_{t}$-term gives:

$$
K^{*} T^{*} K=K^{*} T K \Leftrightarrow K^{*}\left(T^{*}-T\right) K=0,
$$

with $K$ given by (2.20). Since $K$ is invertible by assumption this is equivalent to $(7.2)$.

The vanishing of the $b_{t}$-term gives:

$$
0=i B^{*} K-\bar{\gamma}_{-} B^{*} K^{*} T^{*} K-i A K+\bar{\gamma}_{-} B^{*} K^{*} T K .
$$


Using again the invertibility of $K$ and its explicit form (2.20), this is equivalent to (7.3).

The vanishing of the constant term gives:

$$
\begin{aligned}
0= & \gamma_{-} B^{*} K B-\bar{\gamma}_{-} B^{*} K^{*} A^{*}+i\left|\gamma_{-}\right|^{2} B^{*} K^{*} T^{*} K B \\
& +i C^{*}+\bar{\gamma}_{-} B^{*} K^{*} B-\gamma_{-} A K B-i\left|\gamma_{-}\right|^{2} B^{*} K^{*} T K B-i C,
\end{aligned}
$$

and, since the conditions (7.2) and (7.3) are satisfied, it is equivalent to (7.4).

Conversely, suppose that conditions (7.2)-(7.4) are satisfied. Then, since $\operatorname{Re} \gamma_{-} \neq 0$, it follows that $1+i \gamma_{-} T$ is always invertible. In fact, assuming the contrary, the identity

$$
1+i \gamma_{-} T=i \gamma_{-}\left(\frac{1}{i \gamma_{-}}+T\right)
$$

and the fact that $\gamma_{-} \neq 0$ would imply that also $1 /\left(i \gamma_{-}\right)+T$ is not invertible, i.e. $1 /\left(i \gamma_{-}\right)$should be an element of the spectrum of $T$. But this is a contradiction because $T$ is self-adjoint. Therefore the same arguments as in the first part of the proof show that the right-hand side of $(6.5)$ is identically zero and, since $j_{0}(1)=1$ by assumption, $U_{t}$ is an isometry for every $t$. This proves the statement.

\subsection{The co-isometricity condition}

There are two ways to handle the co-isometries condition for $U$ : one is through the multiplicativity of the forward flow (6.4); another is through the conservativity of the backward flow (6.10) we begin to discuss the latter.

Theorem 7.2. Let $A, B, C$ and $T$ be bounded operators on the initial space $\mathcal{H}_{S}$ then, for the unique solution $U_{t}$ of Eq. (7.1), the following statements are equivalent:

(i) $U_{t}$ is co-isometric, i.e.

$$
U_{t} U_{t}^{*}=1
$$

and both $\left(1+i \gamma_{-} T\right)$ and $\left(1+i \gamma_{-} T^{*}\right)$ are invertible.

(ii) The coefficients $A, B, T$ and $C$ satisfy the relations (7.2)-(7.4) so that Eq. (7.1) takes the form (7.5).

Proof. The co-isometricity condition is equivalent to:

$$
j_{t}(1)=1 \quad \forall t
$$

where $j_{t}$ is given by (6.10). Thus condition (7.7) is equivalent to the fact that the right-hand side of (6.11) vanishes for all $t$. The independence of the basic noises implies then that the coefficients of the various terms on the right-hand side of (6.11) vanish separately. The vanishing of the $b_{t}^{+} b_{t}$-term gives:

$$
T K K^{\prime}=K^{\prime *} K^{*} T^{*}
$$


with $K$ and $K^{\prime}$ defined by (2.20). From (51) and (73), under our assumptions this is equivalent to:

$$
\begin{aligned}
T K K^{\prime} & =T\left(1+i \gamma_{-} T\right)^{-1}\left(1+i \gamma_{-} T^{*}\right)=\left(1+i \gamma_{-} T\right)^{-1} T\left(1+i \gamma_{-} T^{*}\right) \\
& =K^{\prime *} K^{*} T^{*}=\left(1-i \bar{\gamma}_{-} T\right)\left(1-i \bar{\gamma}_{-} T^{*}\right)^{-1} T^{*}=\left(1-i \bar{\gamma}_{-} T\right) T^{*}\left(1-i \bar{\gamma}_{-} T^{*}\right)^{-1},
\end{aligned}
$$

which is equivalent to:

$$
\begin{aligned}
\left(1+i \gamma_{-} T\right)\left(1-i \bar{\gamma}_{-} T\right) T^{*} & =T\left(1+i \gamma_{-} T^{*}\right)\left(1-i \bar{\gamma}_{-} T^{*}\right) \\
& \Leftrightarrow\left(1-\left|\gamma_{-}\right|^{2} T T^{*}\right) T^{*}=T\left(1-\left|\gamma_{-}\right|^{2} T T^{*}\right) .
\end{aligned}
$$

Therefore for any polynomial $P$

$$
P\left(1-\left|\gamma_{-}\right|^{2} T T^{*}\right) T^{*}=T P\left(1-\left|\gamma_{-}\right|^{2} T T^{*}\right) .
$$

This implies, by approximation, that for any measurable function $f$

$$
f\left(1-\left|\gamma_{-}\right|^{2} T T^{*}\right) T^{*}=T f\left(1-\left|\gamma_{-}\right|^{2} T T^{*}\right) .
$$

Since $f$ is arbitrary, one also has

$$
f\left(\left|T^{*}\right|\right) T^{*}=T f\left(\left|T^{*}\right|\right) .
$$

Now, let

$$
T^{*}=\left|T^{*}\right| V
$$

be the polar decomposition of $T^{*}$, so that

$$
T=V^{*}\left|T^{*}\right| \text {. }
$$

Then

$$
f\left(\left|T^{*}\right|\right)\left|T^{*}\right| V=V^{*}\left|T^{*}\right| f\left(\left|T^{*}\right|\right)=V^{*} f\left(\left|T^{*}\right|\right)\left|T^{*}\right| .
$$

Since $f$ is arbitrary this implies that, for any measurable $g$ one has

$$
g\left(\left|T^{*}\right|\right) V=V^{*} g\left(\left|T^{*}\right|\right) .
$$

In particular the choice $g(x)=x$ gives

$$
T^{*}=\left|T^{*}\right| V=V^{*}\left|T^{*}\right|=T .
$$

The $b_{t}^{+}$-term gives, again using $T=T^{*}$ :

$$
-i B-\gamma_{-} T K B+\gamma_{-} T K A^{*}+i K^{*} K^{*} A^{*}=0 .
$$

Since

$$
\begin{aligned}
-i-\gamma_{-} T K & =-i\left(1-i \gamma_{-} T K\right)=-i\left(\frac{1-i \gamma_{-} T}{1+i \gamma_{-} T}\right) \\
& =-i\left(\frac{1+i \gamma_{-} T-i \gamma_{-} T}{1+i \gamma_{-} T}\right)=-i\left(\frac{1}{1+i \gamma_{-} T}\right)=-i K
\end{aligned}
$$

it follows that:

$$
-i B-\gamma_{-} T K B=-i K B
$$


Combining this with $K K^{\prime}=1$ and with (7.9), we find

$$
-i K B+\gamma_{-} T K A^{*}+i A^{*}=0
$$

which implies

$-i K B+\left(\gamma_{-} T K+i\right) A^{*}=-i K B+\left(\gamma_{-} T\left(1+i \gamma_{-} T\right)^{-1}+i\right) A^{*}=-i K B+i K A^{*}=0$, and this too is identically satisfied because $K$ is invertible and $B=A^{*}$ holds by assumption (7.3).

Using the above results, the vanishing of the drift term becomes equivalent to $C=C^{*}$ which is also true by assumption. This completes the proof.

Remark. Notice the asymmetry in the proof of the isometry and of the co-isometry conditions even in the bounded case.

\section{Expression of the Hudson-Parthasarathy Coefficients in Terms of the White Noise Hamiltonian}

Corollary 8.1. Let $A, C$ and $T$ be bounded operators on the initial space $\mathcal{H}_{S}$ satisfying the unitarity conditions (7.2), (7.4). denoting:

$$
\begin{aligned}
S & :=\frac{1-i \bar{\gamma}_{-} T}{1+i \gamma_{-} T}, \\
D^{+} & :=i A \frac{1}{1+i \gamma_{-} T},
\end{aligned}
$$

the causally normally ordered form of the white noise Hamiltonian equation

$$
\partial_{t} U_{t}=-i\left(A b_{t}+A^{*} b_{t}^{+}+b_{t}^{+} T b_{t}+C\right) U_{t}, \quad U_{0}=1,
$$

is

$$
\begin{aligned}
\partial_{t} U_{t}= & S D b_{t}^{+} U_{t}-D^{+} U_{t} b_{t}+\frac{1}{2 \operatorname{Re}\left(\gamma_{-}\right)}(S-1) b_{t}^{+} U_{t} b_{t} \\
& +\left(-\gamma_{-} D^{+} D+i\left|\gamma_{-}\right|^{2} D^{+} T D-i C\right) U_{t},
\end{aligned}
$$

which is equivalent to the stochastic differential equation

$$
\begin{aligned}
d U_{t}= & \left(S D d B_{t}^{+}-D^{*} d B_{t}+\frac{1}{2 \operatorname{Re}\left(\gamma_{-}\right)}(S-1) d N_{t}\right. \\
& \left.+\left(-\gamma_{-} D^{+} D+i\left|\gamma_{-}\right|^{2} D^{+} T D-i C\right) d t\right) U_{t} .
\end{aligned}
$$

Remark. It is known that Eq. (8.5) is the most general unitary stochastic differential equation in the sense of Hudson-Parthasarathy. 
Proof. We have seen that the causally normally ordered form of Eq. (6.1) is, in the notation $(2.20)$ :

$$
\partial_{t} U_{t}=-i b_{t}^{+} K A^{*} U_{t}-i A K U_{t} b_{t}-i T K b_{t}^{+} U_{t} b_{t}+\left(-\gamma_{-} A K A^{*}-i C\right) U_{t} .
$$

Now notice that

$$
\begin{aligned}
-i A^{*}-\gamma_{-} T K A^{*} & =-i\left(1-i \gamma_{-} T K\right) A^{*}=-i\left(1-\frac{i \gamma_{-} T}{1+i \gamma_{-} T}\right) A^{*} \\
& =-i\left(\frac{1+i \gamma_{-} T-i \gamma_{-} T}{1+i \gamma_{-} T}\right) A^{*}=-i\left(\frac{1}{1+i \gamma_{-} T}\right) A^{*}=-i K A^{*} .
\end{aligned}
$$

Let $S$ be defined by (8.1) so that $S:=K K^{*-1}$, then

$$
\begin{aligned}
S-1 & =\frac{1-i \bar{\gamma}_{-} T}{1+i \gamma_{-} T}-1=\frac{1-i \bar{\gamma}_{-} T-1-i \gamma_{-} T}{1+i \gamma_{-} T} \\
& =\frac{-i\left(\bar{\gamma}_{-}+\gamma_{-}\right) T}{1+i \gamma_{-} T}=\frac{-2 i \operatorname{Re}\left(\gamma_{-}\right) T}{1+i \gamma_{-} T}=-2 i \operatorname{Re}\left(\gamma_{-}\right) T K .
\end{aligned}
$$

Therefore, using this, (8.6) becomes

$\partial_{t} U_{t}=-i b_{t}^{+} K A^{*} U_{t}-i A K U_{t} b_{t}+\frac{1}{2 \operatorname{Re}\left(\gamma_{-}\right)}(S-1) b_{t}^{+} U_{t} b_{t}+\left(-\gamma_{-} A K A^{*}-i C\right) U_{t}$

In the notations (8.2) one has:

$$
D=-i \frac{1}{1-i \bar{\gamma}_{-} T} A^{*}
$$

this leads to:

$$
-i K A^{*}=-i \frac{1}{1+i \gamma_{-} T} A^{*}=-i\left(\frac{1-i \bar{\gamma}_{-} T}{1+i \gamma_{-} T}\right) \frac{1}{1-i \bar{\gamma}_{-} T} A^{*}=S D .
$$

For the drift term in (8.8), one has:

$$
\begin{aligned}
-\gamma_{-} A K A^{*} & =-\gamma_{-} A \frac{1}{1+i \gamma_{-} T} A^{*}=-\gamma_{-} A \frac{1-i \bar{\gamma}_{-} T}{\left|1+i \gamma_{-} T\right|^{2}} A^{*} \\
& =\gamma_{-} A \frac{1}{\left|1+i \gamma_{-} T\right|^{2}} A^{*}+i\left|\gamma_{-}\right|^{2} A \frac{T}{\left|1+i \gamma_{-} T\right|^{2}} A^{*} \\
& =-\gamma_{-} D^{+} D+i\left|\gamma_{-}\right|^{2} A \frac{T}{\left|1+i \gamma_{-} T\right|^{2}} A^{*}
\end{aligned}
$$

Then (8.8) is a rewriting of (8.4). Since (8.4) is a normally ordered white noise equation, it is equivalent to the stochastic differential equation (8.5).

Theorem 8.1. Let $j_{t}(x):=U_{t}^{*} x U_{t}$ be the forward inner flow associated to the white noise Hamiltonian equation (6.1) and define:

$$
\sigma(x):=S^{*} x S
$$




$$
\begin{gathered}
\delta^{+}(x):=\sigma(x) D-D x, \\
\delta^{-}(x):=D^{+} \sigma(x)-x D^{+}, \\
\delta_{T}:=i[T, x]=i T x-i x T, \\
\delta_{H}(x):=i[H, x]=i H x-i x H, \\
H:=i \operatorname{Im}\left(\gamma_{-}\right) D^{+} D-\left|\gamma_{-}\right|^{2} D^{+} T D+C, \\
L(x):=2 \operatorname{Re}\left(\gamma_{-}\right)\left(D^{+} \sigma(x) D-\frac{1}{2}\left\{D^{+} D, x\right\}\right)+\delta_{H}(x) .
\end{gathered}
$$

Then, with $K$ given by (2.20), $j_{t}(x)$ satisfies the following causally normally ordered equation:

$$
\partial_{t} j_{t}(x)=b_{t}^{+} j_{t}\left(\delta_{T}\left(K^{*} x K\right)\right) b_{t}+j_{t}\left(\delta^{-}(x)\right) b_{t}+b_{t}^{+} j_{t}\left(\delta^{+}(x)\right)+j_{t}(L(x)),
$$

which is equivalent to the stochastic differential equation:

$$
d j_{t}(x)=j_{t}\left(\delta_{T}\left(K^{*} x K\right)\right) d N_{t}+j_{t}\left(\delta^{-}(x)\right) d B_{t}+j_{t}\left(\delta^{+}(x)\right) d B_{t}^{+}+j_{t}(L(x)) d t .
$$

Proof. Using Eq. (8.4), one has:

$$
\begin{aligned}
\partial_{t} j_{t}(x)= & \partial_{t}\left(U_{t}^{*} x U_{t}\right)=\partial_{t} U_{t}^{*} x U_{t}+U_{t}^{*} x \partial_{t} U_{t} \\
= & {\left[U_{t}^{*} b_{t} D^{+} S^{*}-b_{t}^{+} U_{t}^{*} D+\frac{1}{2 \operatorname{Re}\left(\gamma_{-}\right)} b_{t}^{+} U_{t}^{*} b_{t}\left(S^{*}-1\right)\right.} \\
& \left.+U_{t}^{*}\left(-\bar{\gamma}_{-} D^{+} D-i\left|\gamma_{-}\right|^{2} D^{+} T D+i C\right)\right] x U_{t} \\
& +U_{t}^{*} x\left[S D b_{t}^{+} U_{t}-D^{+} U_{t} b_{t}+\frac{1}{2 \operatorname{Re}\left(\gamma_{-}\right)}(S-1) b_{t}^{+} U_{t} b_{t}\right. \\
& \left.+\left(-\gamma_{-} D^{+} D+i\left|\gamma_{-}\right|^{2} D^{+} T D-i C\right) U_{t}\right],
\end{aligned}
$$

using the identities:

$$
\begin{aligned}
b_{t} U_{T} & =\gamma_{-} S D U_{t}+K U_{t} b_{t}, \\
U_{t}^{*} b_{t}^{+} & =\bar{\gamma}_{-} U_{t}^{*} D^{+} S^{*}+b_{t}^{+} U_{t}^{*} K^{*},
\end{aligned}
$$

one finds

$$
\begin{aligned}
\partial_{t} j_{t}(x)= & U_{t}^{*} D^{+} S^{*} x\left(\gamma_{-} S D U_{t}+K U_{t} b_{t}\right)-b_{t}^{+} U_{t}^{*} D x U_{t} \\
& +\frac{1}{2 \operatorname{Re}\left(\gamma_{-}\right)} b_{t}^{+} U_{t}^{*}\left(S^{*}-1\right) x\left(\gamma_{-} S D U_{t}+K U_{t} b_{t}\right)
\end{aligned}
$$




$$
\begin{aligned}
& +\left(\bar{\gamma}_{-} U_{t}^{*} D^{+} S^{*}+b_{t}^{+} U_{t}^{*} K^{*}\right) x S D U_{t} \\
& -U_{t}^{*} x D^{+} U_{t} b_{t}+\frac{1}{2 \operatorname{Re}\left(\gamma_{-}\right)}\left(\bar{\gamma}_{-} U_{t}^{*} D^{+} S^{*}+b_{t}^{+} U_{t}^{*} K^{*}\right) x(S-1) U_{t} b_{t} \\
& +U_{t}^{*}\left(-\bar{\gamma}_{-} D^{+} D-i\left|\gamma_{-}\right|^{2} D^{+} T D+i C\right) x U_{t} \\
& +U_{t}^{*} x\left(-\gamma_{-} D^{+} D+i\left|\gamma_{-}\right|^{2} D^{+} T D-i C\right) U_{t} \\
& =b_{t}^{+} j_{t}\left(\frac{1}{2 \operatorname{Re}\left(\gamma_{-}\right)}\left(S^{*}-1\right) x K+\frac{1}{2 \operatorname{Re}\left(\gamma_{-}\right)} K^{*} x(S-1)\right) b_{t} \\
& +j_{t}\left(D^{+} S^{*} x K-x D^{+}+\frac{\bar{\gamma}_{-}}{2 \operatorname{Re}\left(\gamma_{-}\right)} D^{+} S^{*} x(S-1)\right) b_{t} \\
& +b_{t}^{+} j_{t}\left(-D x+K^{*} x S D+\frac{\gamma_{-}}{2 \operatorname{Re}\left(\gamma_{-}\right)}\left(S^{*}-1\right) x S D\right) \\
& +j_{t}\left(\gamma_{-} D^{+} S^{*} x S D\right)+j_{t}\left(\bar{\gamma}_{-} D^{+} S^{*} x S D\right) \\
& +j_{t}\left(-\bar{\gamma}_{-} D^{+} D x-i\left|\gamma_{-}\right|^{2} D^{+} T D x+i C x\right) \\
& +j_{t}\left(-\gamma_{-} x D^{+} D+i\left|\gamma_{-}\right|^{2} x D^{+} T D-i x C\right)
\end{aligned}
$$

therefore using (8.7), (8.1), one has:

$$
\begin{aligned}
\partial_{t} j_{t}(x)= & b_{t}^{+} j_{t}\left(i K^{*} T x K-i K^{*} x T K\right) b_{t}+j_{t}\left(D^{+} S^{*} x K-x D^{+}-i \bar{\gamma}_{-} D^{+} S^{*} x T K\right) b_{t} \\
& +b_{t}^{+} j_{t}\left(-D x+K^{*} x S D+i\left(\gamma_{-}\right) K^{*} T x S D\right)+j_{t}\left(2 \operatorname{Re}\left(\gamma_{-}\right) D^{+} S^{*} x S D\right) \\
& +j_{t}\left(-\operatorname{Re}\left(\gamma_{-}\right)\left\{D^{+} D, x\right\}+i \operatorname{Im}\left(\gamma_{-}\right)\left[D^{+} D, x\right]\right)+j_{t}\left(-i\left[\left|\gamma_{-}\right|^{2} D^{+} T D-C, x\right]\right) \\
= & b_{t}^{+} j_{t}\left(i\left[T, K^{*} x K\right]\right) b_{t}+j_{t}\left(D^{+} S^{*} x\left(1-i \bar{\gamma}_{-} T\right) K-x D^{+}\right) b_{t} \\
& +b_{t}^{+} j_{t}\left(-D x+K^{*}\left(1+i\left(\gamma_{-}\right) T\right) x S D\right) \\
& +j_{t}\left(2 \operatorname{Re}\left(\gamma_{-}\right)\left(-\frac{1}{2}\left\{D^{+} D, x\right\}+D^{+} S^{*} x S D\right)\right. \\
& \left.+i \operatorname{Im}\left(\gamma_{-}\right)\left[D^{+} D, x\right]\right)+j_{t}\left(-i\left[\left|\gamma_{-}\right|^{2} D^{+} T D-C, x\right]\right) \\
= & b_{t}^{+} j_{t}\left(i\left[T, K^{*} x K\right]\right) b_{t}+j_{t}\left(\left[D^{+} S^{*}, x\right] S\right) b_{t}+b_{t}^{+} j_{t}\left(S^{-1}[x, S D]\right) \\
& +j_{t}\left(2 \operatorname{Re}\left(\gamma_{-}\right)\left(-\frac{1}{2}\left\{D^{+} D, x\right\}+D^{+} S^{*} x S D\right)+i \operatorname{Im}\left(\gamma_{-}\right)\left[D^{+} D, x\right]\right) \\
& +j_{t}\left(-i\left[\left|\gamma_{-}\right|^{2} D^{+} T D-C, x\right]\right) .
\end{aligned}
$$

In the notations (8.9) and (8.10) we see that:

$$
\begin{aligned}
\delta^{+}(x y) & =\sigma(x y) D-D x y=\sigma(x) \sigma(y) D-D x y \\
& =\sigma(x)[\sigma(y) D-D y]+\sigma(x) D y-D x y \\
& =\sigma(x)[\sigma(y) D-D y]+[\sigma(x) D-D x] y=\delta^{+}(x) y+\sigma(x) \delta^{+}(y) .
\end{aligned}
$$


Thus $\delta^{+}$is a right- $\sigma$-derivation. Similarly $\delta^{-}$, defined by (8.11) is a left- $\sigma$-derivation. In fact

$$
\begin{aligned}
\delta^{-}(x y) & =D^{+} \sigma(x y)-x y D^{+}=D^{+} \sigma(x) \sigma(y)-x y D^{+} \\
& =\left[D^{+} \sigma(y)-y D^{+}\right] \sigma(y)+x D^{+} \sigma(y)-x y D^{+}=\delta^{-}(x) \sigma(y)+x \delta^{-}(y) .
\end{aligned}
$$

It follows that:

$$
\begin{aligned}
\partial_{t} j_{t}(x)= & b_{t}^{+} j_{t}\left(i\left[T, K^{*} x K\right]\right) b_{t}+j_{t}\left(D^{+} \sigma(x)-x D^{+}\right) b_{t}+b_{t}^{+} j_{t}(\sigma(x) D-D x) \\
& +j_{t}\left(2 \operatorname{Re}\left(\gamma_{-}\right)\left(-\frac{1}{2}\left\{D^{+} D, x\right\}+D^{+} \sigma(x) D\right)+i \operatorname{Im}\left(\gamma_{-}\right)\left[D^{+} D, x\right]\right) \\
& +j_{t}\left(-i\left[\left|\gamma_{-}\right|^{2} D^{+} T D-C, x\right]\right)
\end{aligned}
$$

which, in the notations introduced above, coincides with (8.16).

Remark 8.1. Combining the results of Theorems 8.1 and 5.1 we obtain the expression of the structure maps as functions of the original Hamiltonian coefficients. In fact, in the notations of Theorem 8.1 , these are given by:

$$
\begin{gathered}
\delta_{2}(x):=\delta_{T}\left(K^{*} x K\right), \\
\delta_{1}(x):=\delta^{+}(x), \\
\delta_{-1}(x):=\delta^{-}(x), \\
\delta_{0}(y):=2 \operatorname{Re}\left(\gamma_{-}\right)\left(-\frac{1}{2}\left\{D^{+} D, x\right\}+D^{+} \sigma(x) D\right)+\delta_{H}(x) .
\end{gathered}
$$

\section{References}

1. L. Accardi, Y. G. Lu and I. Volovich, Quantum Theory and Its Stochastic Limit (Springer-Verlag, 2002).

2. L. Accardi, Y. G. Lu and I. Volovich, The stochastic sector of quantum field theory, Mat. Z. (1994), Volterra preprint No. 138 (1993).

3. L. Accardi, Y. G. Lu and I. Volovich, Nonlinear extensions of classical and quantum stochastic calculus and essentially infinite dimensional analysis, in Probability Towards 2000, Columbia University, New York, 2-6 October 1995, Springer LN in Statistics, Vol. 128, eds. L. Accardi and C. Heyde (Springer, 1998), pp. 1-33.

4. L. Accardi, Y. G. Lu and N. Obata, Towards a non-linear extension of stochastic calculus, in Publications of the Research Institute for Mathematical Sciences, Kyoto, RIMS Kokyuroku 957, ed. N. Obata (1996), pp. 1-15.

5. L. Accardi, Y. G. Lu and I. Volovich, White noise approach to classical and quantum stochastic calculi, Lecture Notes of the Volterra-CIRM International School with the same title, Trento, Italy, 1999, Volterra Preprint No. 375 July (1999).

6. L. Accardi, W. Ayed and H. Ouerdiane, White Noise Approach to Stochastic Integration, talk given (by W.A.) at the Colloque International de Mathematiques, Analyse et Probabilites, 20-25 Octobre 2003, Hammamet, (Tunisie), submitted to Random Operators and Stochastic Equations, Preprint Volterra n. 573 (2004). 
7. L. Accardi and A. Boukas, Unitarity conditions for the renormalized square of white noise, in Trends in Contemporary Infinite Dimensional Analysis and Quantum Probability, Natural and Mathematical Sciences Series, Vol. 3 (Italian School of East Asian Studies, 2000), 7-36 Preprint Volterra No. 405 (2000).

8. L. Accardi and A. Boukas, Unitarity conditions for the renormalized square of white noise, Inf. Dim. Anal. Quantum Probab. Rel. Topics 6 (2003) 197-222.

9. L. Accardi, F. Fagnola and Quaegebeur, A representation free quantum stochastic calculus, J. Funct. Anal. 104 (1992) 149-197.

10. L. Accardi, U. Franz and M. Skeide, Renormalized squares of white noise and other non-Gaussian noises as Levy processes on real Lie algebras, Comm. Math. Phys. 228 (2002) 123-150.

11. L. Accardi, A. Boukas and U. Franz, Renormalized powers of quantum white noise, Inf. Dim. Anal. Quantum Probab. Rel. Topics 9 (2005) 129-147.

12. J. Gough, Noncommutative Ito and Stratonovich noise and stochastic evolutions, Theor. Math. Phys. 113 (1997) 276-284.

13. J. Gough, Noncommutative Markov approximations, Dokl. Math. 64 (2001) 112-116 [Dokl. Akad. Nauk 379 730-734 (2001), in Russian].

14. T. Hida, Selected Papers of Takeyuki Hida, eds. L. Accardi, H. H. Kuo, N. Obata, K. Saitô, Si Si and L. Streit (World Scientific, 2001).

15. T. Hida, Brownian Motion, Applications of Mathematics, Vol. 11, translated from the Japanese by the author and T. P. Speed (Springer-Verlag, 1980).

16. M. Evans and R. L. Hudson, Multidimensional quantum diffusions, in Quantum Probability and Applications III, Springer Lecture Note in Mathematics, Vol. 1303, eds. L. Accardi, W. von Waldenfels (Springer, 1988), pp. 68-89.

17. R. L. Hudson and J. M. Lindsay, On characterizing quantum stochastic evolutions, Math. Proc. Cambridge Phil. Soc. 102 (1987) 363-370.

18. R. L. Hudson and K. R. Parthasarathy, Quantum Ito's formula and stochastic evolutions, Comm. Math. Phys. 93 (1984) 301-323.

19. H. H. Kuo, White Noise Distribution Theory, Probability and Stochastics Series (CRC Press, 1996).

20. K. R. Parthasarathy, An Introduction to Quantum Stochastic Calculus, Monographs in Mathematics, Vol. 85 (Birkhäuser-Verlag, 1992).

21. Y. Kosaku, Functional Analysis, Classics in Mathematics, reprint of the 6th edn. (Springer-Verlag, 1995). 